\title{
Next-to-leading order QCD analysis of the spin structure function $g_{1}$
}

B. Adeva, ${ }^{18}$ T. Akdogan, ${ }^{2}$ E. Arik, ${ }^{2}$ B. Badelek, ${ }^{21,23}$ G. Bardin,,${ }^{17, a}$ G. Baum,,${ }^{1}$ P. Berglund ${ }^{8}$ L. Betev, ${ }^{13}$ R. Birsa, ${ }^{20}$ N. de Botton, ${ }^{17}$ F. Bradamante, ${ }^{20}$ A. Bravar, ${ }^{11}$ A. Bressan ${ }^{20, b}$ S. Bültmann, ${ }^{1, \mathrm{c}}$ E. Burtin, ${ }^{17}$ C. Cavata, ${ }^{17}$ D. Crabb, ${ }^{22}$

J. Cranshaw, ${ }^{20}$ T. Çuhadai, ${ }^{2,15}$ S. Dalla Torre ${ }^{20}{ }^{17}$ R. van Dantzig, ${ }^{15}$ B. Derro, ${ }^{4}$ A. Deshpande,${ }^{24}$ S. Dhawan, ${ }^{24}$ C. Dulya ${ }^{15,4, d}$ S. Eichblatt, ${ }^{\mathrm{e}}$ D. Fasching, ${ }^{16, \mathrm{f}}$ F. Feinstein, ${ }^{17}$ C. Fernandez, ${ }^{18,9}$ S. Forthmann, ${ }^{7}$ B. Frois, ${ }^{17}$ A. Gallas, ${ }^{18}$ J. A. Garzon, ${ }^{18,9}$ H. Gilly, ${ }^{6}$ M. Giorgi, ${ }^{20}$ E. von Goeler, ${ }^{\mathrm{g}}$ S. Goertz, ${ }^{3}$ G. Gracia, ${ }^{18, \mathrm{~h}}$ N. de Groot,,${ }^{15, \mathrm{i}}$ M. Grosse Perdekamp, ${ }^{24, \mathrm{j}} \mathrm{K}$. Haft, ${ }^{13}$ D. von Harrach, ${ }^{11}$ T. Hasegawa,${ }^{14, \mathrm{k}}$ P. Hautle,${ }^{5,1}$ N. Hayashi,,${ }^{14, \mathrm{~m}}$ C. A. Heusch,${ }^{5, \mathrm{n}}$ N. Horikawa,${ }^{14}$ V. W. Hughes,${ }^{24}$ G. Igo, ${ }^{4}$ S. Ishimoto, ${ }^{14,0}$ T. Iwata, ${ }^{14}$ E. M. Kabuß, ${ }^{11}$ T. Kageya, ${ }^{14, p}$ A. Karev ${ }^{10}$ H. J. Kessler ${ }^{6, q}$ T. J. Ketel, ${ }^{15}$ J. Kiryluk, ${ }^{23}$ Yu. Kisselev, ${ }^{10}$ D. Krämer, ${ }^{1}$ V. Krivokhijine, ${ }^{10}$ W. Kröger,${ }^{5, \mathrm{n}}$ V. Kukhtin, ${ }^{10}$ K. Kurek, ${ }^{23}$ J. Kyynäräinen, ${ }^{1,8}$

M. Lamanna, ${ }^{20}$ U. Landgraf, ${ }^{6}$ J. M. Le Goff, ${ }^{17}$ F. Lehar, ${ }^{17}$ A. de Lesquen, ${ }^{17}$ J. Lichtenstadt, ${ }^{19}$ M. Litmaath,,${ }^{15, b}$ A. Magnon, ${ }^{17}$ G. K. Mallot, ${ }^{11, b}$ F. Marie,${ }^{17}$ A. Martin, ${ }^{20}$ J. Martino, ${ }^{17}$ T. Matsuda,${ }^{14, k}$ B. Mayes, ${ }^{9}$ J. S. McCarthy, ${ }^{22}$ K. Medved,${ }^{10}$

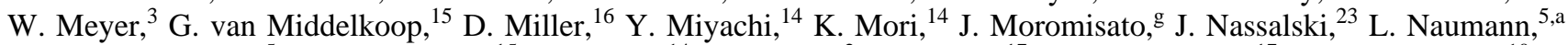

T. O. Niinikoski, ${ }^{5}$ J. E. J. Oberski, ${ }^{15}$ A. Ogawa, ${ }^{14, \mathrm{r}}$ C. Ozben, ${ }^{2}$ H. Pereira ${ }^{17}$ F. Perrot-Kunne, ${ }^{17}$ D. Peshekhonov, ${ }^{10}$ R. Piegaia,${ }^{5, \mathrm{~s}}$ L. Pinsky, ${ }^{9}$ S. Platchkov, ${ }^{17}$ M. Plo, ${ }^{18}$ D. Pose,${ }^{10}$ H. Postma,${ }^{15}$ J. Pretz,${ }^{11, t}$ R. Puntaferro, ${ }^{20}$ T. Pussieux ${ }^{17}$

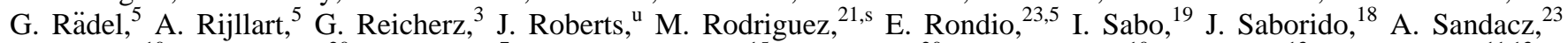
I. Savin, ${ }^{10}$ P. Schiavon, ${ }^{20}$ A. Schiller, ${ }^{7}$ E. P. Sichtermann, ${ }^{15}$ F. Simeoni,${ }^{20}$ G. I. Smirnov ${ }^{10}$ A. Staude, ${ }^{13}$ A. Steinmetz,,${ }^{11,13}$ U. Stiegler, ${ }^{5}$ H. Stuhrmann, ${ }^{7}$ M. Szleper ${ }^{23}$ F. Tessarotto, ${ }^{20}$ D. Thers,,${ }^{17}$ W. Tlaczala, ${ }^{23, v}$ A. Tripet, ${ }^{1}$ G. Unel, ${ }^{2}$ M. Velasco, ${ }^{16, b}$ J. Vogt, ${ }^{13}$ R. Voss, ${ }^{5}$ C. Whitten, ${ }^{4}$ R. Windmolders, ${ }^{12}$ R. Willumeit, ${ }^{7}$ W. Wislicki, ${ }^{23}$ A. Witzmann, ${ }^{6, w}$ J. Ylöstalo, ${ }^{8}$ A. M. Zanetti, ${ }^{20} \mathrm{~K}$. Zaremba, ${ }^{23, v}$ and J. Zhao ${ }^{7, x}$

(Spin Muon Collaboration)

${ }^{1}$ University of Bielefeld, Physics Department, D-33501 Bielefeld, Germany

${ }^{2}$ Bogaziçi University and Istanbul Technical University, Istanbul, Turkey

${ }^{3}$ University of Bochum, Physics Department, D-44780 Bochum, Germany

${ }^{4}$ University of California, Department of Physics, Los Angeles, California 90024 ${ }^{5}$ CERN, 1211 Geneva 23, Switzerland

${ }^{6}$ University of Freiburg, Physics Department, D-79104 Freiburg, Germany ${ }^{7}$ GKSS, D-21494 Geesthacht, Germany

${ }^{8}$ Helsinki University of Technology, Low Temperature Laboratory and Institute of Particle Physics Technology, Espoo, Finland

${ }^{9}$ University of Houston, Department of Physics, Houston, Texas 77204-5506 ${ }^{10} J I N R$, Dubna, RU-141980 Dubna, Russia

${ }^{11}$ University of Mainz, Institute for Nuclear Physics, D-55099 Mainz, Germany

${ }^{12}$ University of Mons, Faculty of Science, 7000 Mons, Belgium

${ }^{13}$ University of Munich, Physics Department, D-80799 Munich, Germany

${ }^{14}$ Nagoya University, CIRSE and Department of Physics, Furo-Cho, Chikusa-Ku, 464 Nagoya, Japan

${ }^{15}$ NIKHEF, Delft University of Technology, FOM and Free University, 1009 AJ Amsterdam, The Netherlands

${ }^{16}$ Northwestern University, Department of Physics, Evanston, Illinois 60208

${ }^{17}$ CEA Saclay, DAPNIA, 91191 Gif-sur-Yvette, France

${ }^{18}$ University of Santiago, Department of Particle Physics, 15706 Santiago de Compostela, Spain

${ }^{19}$ Tel Aviv University, School of Physics, 69978 Tel Aviv, Israel

${ }^{20}$ INFN Trieste and University of Trieste, Department of Physics, 34127 Trieste, Italy

${ }^{21}$ Uppsala University, Department of Radiation Sciences, 75121 Uppsala, Sweden

${ }^{22}$ University of Virginia, Department of Physics, Charlottesville, Virginia 22901

${ }^{23}$ Soltan Institute for Nuclear Studies and Warsaw University, 00681 Warsaw, Poland

${ }^{24}$ Yale University, Department of Physics, New Haven, Connecticut 06511

(Received 18 May 1998; published 19 October 1998)

We present a next-to-leading order QCD analysis of the presently available data on the spin structure function $g_{1}$ including the final data from the Spin Muon Collaboration. We present results for the first moments of the proton, deuteron, and neutron structure functions, and determine singlet and nonsinglet parton distributions in two factorization schemes. We also test the Bjorken sum rule and find agreement with the theoretical prediction at the level of 10\%. [S0556-2821(98)07117-3]

PACS number(s): 13.60.Hb, 13.88.+e

\section{INTRODUCTION}

We present a perturbative QCD (PQCD) analysis in nextto-leading order (NLO) of the world data on polarized lepton-nucleon deep inelastic scattering (DIS). The data used in this analysis include the final results [1] presented by the Spin Muon Collaboration (SMC). From the world data we determine the first moments of the polarized structure functions.

The accuracy of the experimental data on the polarized 
structure function $g_{1}(x)$ has improved significantly in the past few years. All experiments have confirmed the small values of the first moments of $g_{1}$ of the nucleon, thus confirming the violation of the Ellis-Jaffe spin sum rule [2] and the small contribution of quark spins to the nucleon spin $\left(a_{0}\right.$ in the naive quark parton model). Motivated by the availability of accurate experimental data, theoretical tools to analyze them have been advanced, e.g., NLO calculations in PQCD for the spin structure functions. The nucleon spin can now be separated into some of its components in the framework of PQCD. Of special interest is the role played by the polarized gluon distribution. It has been suggested [3] that if the polarized gluon distribution is found to be significant, it could explain the small value of the quark contribution to the proton spin.

The Bjorken sum rule [4] is a relation between the first moments of the spin structure functions of proton and neutron. It is a fundamental result of QCD first derived using current algebra. Most experimental efforts in the past have been oriented towards the direct confirmation of this relation. The determinations of the first moments from the experimental data depended on extrapolations due to the limited kinematic range of the experiments. In this paper we address this issue within the framework of PQCD: we first present a PQCD analysis of the world data assuming the Bjorken sum rule to be valid, and discuss the uncertainties in the analysis and their origins. We then release the Bjorken sum constraint and check if the available data and the theoretical framework of PQCD allow a test of the Bjorken sum rule.

A number of theoretical papers have been published on

\footnotetext{
${ }^{\mathrm{a}}$ Deceased.

${ }^{\mathrm{b}}$ Now at CERN, 1211 Geneva 23, Switzerland.

${ }^{\mathrm{c}}$ Now at University of Virginia, Department of Physics, Charlottesville, VA 22901.

${ }^{\mathrm{d}}$ Now at CIEMAT, Avda Complutense 22, 28040, Madrid, Spain.

${ }^{\mathrm{e} N o w}$ at Fermi National Accelerator Laboratory, Batavia, IL 60510.

${ }^{\mathrm{f}}$ Now at University of Wisconsin.

${ }^{\text {g}}$ Permanent address: Northeastern University, Department of Physics, Boston, MA 02115.

${ }^{\mathrm{h}}$ Now at NIKHEF P.O.B. 41882, 1009 DB Amsterdam, The Netherlands.

${ }^{\mathrm{i}}$ Now at SLAC, Stanford, CA 94309.

${ }^{\mathrm{j}}$ Now at University of Mainz, Institute of Nuclear Physics, 55099, Germany.

${ }^{k}$ Permanent address: Miyazaki University, Faculty of Engineering, 889-21 Miyazaki-Shi, Japan.

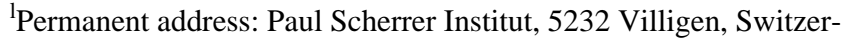
land.

${ }^{m}$ Permanent address: The Institute of Physical and Chemical Research (RIKEN), Wako 351-01, Japan.

${ }^{\mathrm{n}}$ Permanent address: University of California, Institute of Particle Physics, Santa Cruz, CA 95064.

${ }^{\circ}$ Permanent address: KEK, Tsukuba-Shi, 305 Ibaraki-Ken, Japan.

${ }^{\mathrm{p}}$ Now at University of Michigan, Ann Arbor MI 48109.

${ }^{\mathrm{q}}$ Now at SBC Warburg Dillon Read, CH-4002 Basel, Switzerland.

${ }^{\mathrm{r}}$ Now at Penn. State University, 303 Osmond Lab, University
}

this topic over the last few years [5-8]. The E154 Collaboration has recently presented their $\mathrm{PQCD}$ analysis of the data [9]. The SMC has published results in which the PQCD analysis was used to evaluate the first moments $\Gamma_{1}^{p, d, n}$ at a fixed $Q^{2}$ [10-12], but a detailed description of the procedure of the PQCD analysis was not given. We do that in this paper.

In the PQCD analysis, apart from the published data from other collaborations at CERN, SLAC, and DESY, we use a new and final set of data [1] from SMC which includes improved values of $g_{1}$ at low $x$ obtained by requiring the presence of a high energy hadron in the final state. In addition, an improved value of the beam polarization (with respect to our previous publications [10-12]) was used in the evaluation of the asymmetries. We study the impact of each experimental data set and the sources of theoretical uncertainties on the first moments of the spin structure functions and on the polarized parton distributions.

In Sec. II, after a brief overview of the theoretical framework needed for the PQCD analysis, we describe the method used. We performed this analysis using two different mathematical approaches and computer codes. With the improved data available today, we determine the polarized parton distributions and study their stability. A comparison of results obtained in the two programs allows us to do this. We discuss the main features of the two programs used for calculating the $Q^{2}$ evolution emphasizing their differences and similarities, and compare the results obtained. The choice of the factorization scheme has been discussed extensively in Refs. $[6,15]$. It has been shown analytically that the choice is arbitrary, and that one can translate results from one scheme to the other. We chose two widely used schemes in the field of polarized DIS and present results based on world data in those two schemes. Recently, Altarelli et al. [8] have presented results on the determination of $\alpha_{s}$ using the PQCD analysis of the spin structure function data. A publication [9] by the E154 Collaboration has also indicated that their analysis showed sensitivity to the value of $\alpha_{s}$. We present our result and comment on it. Towards the end of Sec. II we discuss in detail the experimental systematic and theoretical sources that contribute to the total uncertainty in the polarized parton distribution functions (PDFs).

Section III discusses the results, namely the first moments of the spin structure functions, the quark and gluon parton distribution functions, and the evaluation of the Bjorken sum

\footnotetext{
Park, PA 16802.

${ }^{\text {s} P e r m a n e n t ~ a d d r e s s: ~ U n i v e r s i t y ~ o f ~ B u e n o s ~ A i r e s, ~ P h y s i c s ~ D e p a r t-~}$ ment, 1428 Buenos Aires, Argentina.

${ }^{t}$ Now at Physics Department, Yale University, New Haven CT 06520.

uPermanent address: Rice University, Bonner Laboratory, Houston, TX 77251-1892.

${ }^{\mathrm{v}}$ Permanent address: Warsaw University of Technology, Warsaw, Poland.

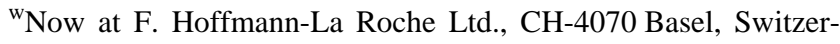
land.

${ }^{x}$ Now at Los Alamos National Laboratory, Los Alamos, NM 87545.
} 
rule. We present two evaluations of the Bjorken sum rule: one from the QCD fit in NLO and another from a fit restricted to the nonsinglet part of the spin structure function.

\section{THE QCD ANALYSIS-PROCEDURE AND UNCERTAINTY ESTIMATION}

\section{A. Introduction: Experimental measurement of $g_{1}$}

In polarized DIS experiments the asymmetry, $A_{\|}$, of the cross sections for parallel and antiparallel orientations of the beam and target spins

$$
A_{\|}=\frac{\sigma^{\uparrow \downarrow}-\sigma^{\uparrow \uparrow}}{\sigma^{\uparrow \downarrow}+\sigma^{\uparrow \uparrow}}
$$

is measured. The evaluation of the asymmetry $A_{\|}$requires knowledge of the incident beam and target polarizations, and of the dilution factor which accounts for the fact that only a fraction of the target nucleons is polarizable. The asymmetry $A_{\|}$and the spin-dependent structure function $g_{1}$ are related to the virtual photon-nucleon asymmetries $A_{1}$ and $A_{2}$ [16] by

$$
A_{\|}=D\left(A_{1}+\eta A_{2}\right), \quad g_{1}=\frac{F_{2}}{2 x(1+R)}\left(A_{1}+\gamma A_{2}\right),
$$

in which the factors $\eta$ and $\gamma$ depend only on kinematic variables and on the nucleon mass, while the depolarization factor $D$ depends on kinematic variables and the ratio of total photoabsorption cross sections for longitudinally and transversely polarized virtual photons $R=\sigma_{L} / \sigma_{T}$. The structure function $g_{1}$ is computed using Eq. (2) and parametrizations for $F_{2}$ [1] and $R$. For $x<0.12$ a parametrization of $R$ based on the data from Ref. [13] was used, while for $x>0.12$ the parametrization in Ref. [14] was used. For other experimental aspects of the $g_{1}$ measurement see Refs. [1,12].

In the PQCD analysis presented in this paper we use the final SMC proton and deuteron data from Ref. [1] with $Q^{2}$ $>1 \mathrm{GeV}^{2}$, the proton data from the European Muon Collaboration (EMC) [17], the proton and deuteron data from the E143 Collaboration [18-20], and the neutron data from the E142 [21], E154 [22], and HERMES [23] Collaborations.

As in our previous publications [10], we assume that the deuteron structure function $g_{1}^{d}$ is related to the proton and neutron structure functions $g_{1}^{p}$ and $g_{1}^{n}$ by

$$
g_{1}^{p}+g_{1}^{n}=\frac{2 g_{1}^{d}}{\left[1-(3 / 2) \omega_{D}\right]},
$$

where $\omega_{D}=0.05 \pm 0.01$ is the $D$-wave state probability in the deuteron.

\section{B. Theoretical framework}

The structure function $g_{1}$ is related to the polarized quark and gluon distributions through

$$
\begin{aligned}
g_{1}(x, t)= & \frac{1}{2}\left\langle e^{2}\right\rangle \int_{x}^{1} \frac{d y}{y}\left[C_{S}^{q}\left(\frac{x}{y}, \alpha_{s}(t)\right) \Delta \Sigma(y, t)\right. \\
& +2 n_{f} C^{g}\left(\frac{x}{y}, \alpha_{s}(t)\right) \Delta g(y, t) \\
& \left.+C_{\mathrm{NS}}^{q}\left(\frac{x}{y}, \alpha_{s}(t)\right) \Delta q_{\mathrm{NS}}(y, t)\right]
\end{aligned}
$$

where $\left\langle e^{2}\right\rangle=n_{f}^{-1} \sum_{k=1}^{n_{f}} e_{k}^{2}$ is the average squared quark charge, $t=\ln \left(Q^{2} / \Lambda^{2}\right)$ where $\Lambda$ is the QCD scale parameter, $\Delta \Sigma$ and $\Delta q_{\mathrm{NS}}$ are the singlet and nonsinglet polarized quark distributions

$$
\Delta \Sigma(x, t)=\sum_{i=1}^{n_{f}} \Delta q_{i}(x, t),
$$

$$
\Delta q_{\mathrm{NS}}(x, t)=\sum_{i=1}^{n_{f}}\left(e_{i}^{2} /\left\langle e^{2}\right\rangle-1\right) \Delta q_{i}(x, t),
$$

and $C_{S, \mathrm{NS}}^{q}\left(\alpha_{s}(t)\right)$ and $C^{g}\left(\alpha_{s}(t)\right)$ are the quark and gluon coefficient functions. The $x$ and $Q^{2}$ dependence of the polarized quark and gluon distributions is given by the Dokshitzer-Gribov-Lipatov-Altarelli-Parisi (DGLAP) equations [24]

$$
\begin{aligned}
\frac{d}{d t} \Delta \Sigma(x, t)= & \frac{\alpha_{s}(t)}{2 \pi} \int_{x}^{1} \frac{d y}{y}\left[P_{q q}^{S}\left(\frac{x}{y}, \alpha_{s}(t)\right) \Delta \Sigma(y, t)\right. \\
& \left.+2 n_{f} P_{q g}\left(\frac{x}{y}, \alpha_{s}(t)\right) \Delta g(y, t)\right], \\
\frac{d}{d t} \Delta g(x, t)= & \frac{\alpha_{s}(t)}{2 \pi} \int_{x}^{1} \frac{d y}{y}\left\{P_{g q}\left[\frac{x}{y}, \alpha_{s}(t)\right] \Delta \Sigma(y, t)\right. \\
& \left.+P_{g g}\left[\frac{x}{y}, \alpha_{s}(t)\right] \Delta g(y, t)\right\}, \\
\frac{d}{d t} \Delta q_{\mathrm{NS}}(x, t)= & \frac{\alpha_{s}(t)}{2 \pi} \int_{x}^{1}\left[\frac{d y}{y}\right] P_{q q}^{\mathrm{NS}}\left[\frac{x}{y}, \alpha_{s}(t)\right] \Delta q_{\mathrm{NS}}(y, t),
\end{aligned}
$$

where $P_{i j}$ are polarized splitting functions.

The full set of coefficient functions [25] and splitting functions [26] has been computed up to next-to-leading order in $\alpha_{s}$. At next-to-leading order the splitting functions, the coefficient functions, and in general the parton distributions depend on the renormalization and factorization schemes, while the physical observables, such as $g_{1}$, remain scheme independent. Parton distributions in different schemes can be different but they are related to each other by well-defined transformations [15].

Two widely used schemes in the PQCD analysis of the spin structure function data are the modified minimal subtraction $(\overline{\mathrm{MS}})$ scheme [27] and the Adler-Bardeen (AB) [6] scheme which is a modified $\overline{\mathrm{MS}}$ scheme. In the $\overline{\mathrm{MS}}$ scheme the first moment of the gluon coefficient function $C^{g}$ is equal to zero, which implies that the gluon density $\Delta g\left(x, Q^{2}\right)$ does not contribute to the integral $\Gamma_{1}=\int_{0}^{1} g_{1}(x) d x$ [see Eq. (4)]. 
In the $\mathrm{AB}$ scheme the axial anomaly $\left[\sim \alpha_{s}\left(Q^{2}\right) \Delta g\left(Q^{2}\right)\right]$ contributes explicitly to $\Gamma_{1}$. The first moments of the singlet quark distribution in the two schemes differ by an amount proportional to $\alpha_{s} \Delta g$ :

$$
\Delta \Sigma_{\overline{\mathrm{MS}}}\left(Q^{2}\right)=\Delta \Sigma_{\mathrm{AB}}-n_{f} \frac{\alpha_{s}\left(Q^{2}\right)}{2 \pi} \Delta g\left(Q^{2}\right),
$$

where $\Delta g\left(Q^{2}\right)$ is the value of $\Delta g$ that one obtains in an analysis performed in the $\mathrm{AB}$ scheme. Since at leading order the first moment of the polarized gluon distribution behaves as $1 / \alpha_{s}$, the scheme dependence in Eq. (9) persists at all $Q^{2}$ and is potentially large if the first moment of the gluon distribution is large [3].

\section{Method of QCD analysis}

Polarized parton distributions are extracted from experimental structure function data in the following way. One needs an initial functional form for the parton distributions at an initial $Q^{2}=Q_{i}^{2}$. It needs to be flexible enough to allow for the description of the low $x$ as well as the high $x$ behavior of the data and to connect the high and low $x$ behaviors with a minimal number of free parameters. In this spirit we parametrize the initial polarized parton distributions at a starting $Q^{2}=Q_{i}^{2}$ as

$$
\Delta f\left(x, Q^{2}\right)=N\left(\alpha_{f}, \beta_{f}, a_{f}\right) \eta_{f} x^{\alpha_{f}}(1-x)^{\beta_{f}\left(1+a_{f} x\right),}
$$

where $N(\alpha, \beta, a)$ is fixed by the normalization condition

$$
N(\alpha, \beta, a) \int_{0}^{1} x^{\alpha}(1-x)^{\beta}(1+a x) d x=1,
$$

and $\Delta f$ denotes $\Delta \Sigma, \Delta q_{\mathrm{NS}}$, or $\Delta g$. With this normalization the parameters $\eta_{g}, \eta_{\mathrm{NS}}$, and $\eta_{S}$ are the first moments of the gluon, the nonsinglet quark and the singlet quark distributions at the starting scale, respectively. We evolve the initial parton distributions to the $Q^{2}$ of the data points using Eqs. (6)-(8) and evaluate $g_{1}$ with Eq. (4). We determine a $\chi^{2}$ using this calculated $g_{1}, g_{1}^{\text {calc }}\left(x, Q^{2}\right)$, the measured $g_{1}^{\text {data }}\left(x, Q^{2}\right)$, and its statistical uncertainty $\delta_{\text {stat }} g_{1}^{\text {data }}\left(x, Q^{2}\right)$ as

$$
\chi^{2}=\sum_{i=1}^{n} \frac{\left[g_{1}^{\text {calc }}\left(x, Q^{2}\right)-g_{1}^{\text {data }}\left(x, Q^{2}\right)\right]^{2}}{\left[\delta_{\text {stat }} g_{1}^{\text {data }}\left(x, Q^{2}\right)\right]^{2}} .
$$

Here $n$ stands for the number of experimental data points used in the PQCD fit. We minimize this $\chi^{2}$ by changing the initial parton distribution coefficients $\eta_{f}, \alpha_{f}, \beta_{f}$, and $a_{f}$ to get the best fit parton distribution at the initial $Q_{i}^{2}$. Only statistical errors on the data were used in the fit. Various systematic uncertainties, being correlated, had to be handled separately and will be discussed in Sec. II G. Unless otherwise mentioned we chose the initial scale, $Q_{i}^{2}=1 \mathrm{GeV}^{2}$. Since most of the experimental data lie in the range $1<Q^{2}$ $<10 \mathrm{GeV}^{2}$, when it was relevant to study the $Q_{i}^{2}$ dependence of a result, we have done so using $Q_{i}^{2}=10 \mathrm{GeV}^{2}$ as the upper limit for the initial scale. The normalization of the non-
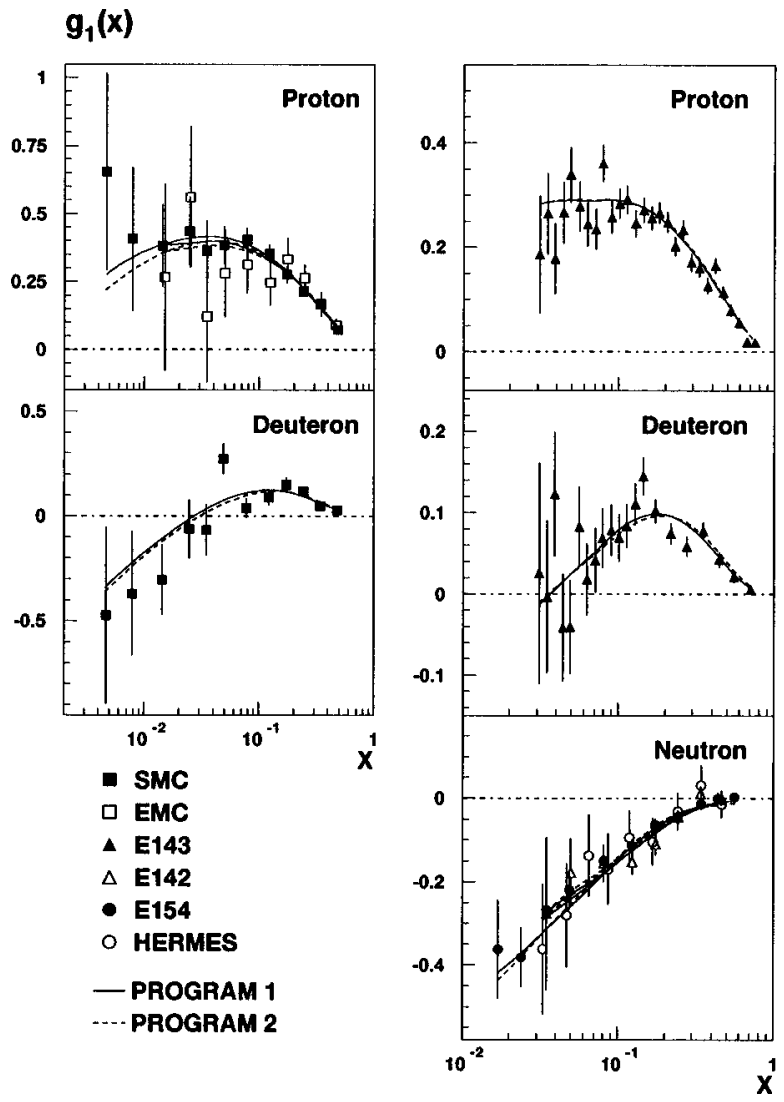

FIG. 1. Comparison of the two programs in the $\overline{\mathrm{MS}}$ Scheme. Data on $g_{1}^{p, d, n}$ from CERN experiments (left column), SLAC, and DESY experiments (right column) are shown at their measured $Q^{2}$ with their statistical errors. The results of the QCD fits using the two programs at the measured $Q^{2}$ of the data are shown by continuous and dashed lines in each plot. Note that some of the fits for SLAC and DESY experiments (right column) are almost indistinguishable.

singlet quark densities $\eta_{\mathrm{NS}}^{p, n}$ are fixed using the neutron and hyperon $\beta$ decay constants and assuming $\mathrm{SU}(3)$ flavor symmetry $\eta_{\mathrm{NS}}^{p, n}=( \pm) \frac{3}{4}\left(g_{A} / g_{V}\right)+\frac{1}{4} a_{8}$. We use $\left|g_{A} / g_{V}\right|=F+D$ $=1.2601 \pm 0.0025$ [28] and $F / D=0.575 \pm 0.016$ [29]. In the analyses in this paper which test the Bjorken sum rule the value of $g_{A} / g_{V}$ will be made a free parameter in the fit. In order to be able to estimate the effect of the yet unknown higher-than-NLO corrections to this analysis, the factorization scale $M^{2}$ and the renormalization scale $\mu^{2}$ in this analysis were taken to be of the form $M^{2}=k_{1} Q^{2}$ and $\mu^{2}=k_{2} Q^{2}$ with $k_{1}=k_{2}=1$ for the standard fit. The variation in the factors $k_{1,2}$ and its role in the uncertainty estimation is discussed in Sec. II G. The value of $\alpha_{s}\left(M_{Z}^{2}\right)=0.118 \pm 0.003$ [28] was used in the analysis. Some tests were done to study the determination of $\alpha_{s}\left(Q^{2}\right)$ from the spin structure function data. They will be discussed in Sec. II F.

\section{Comparison of two QCD evolution programs}

The PQCD analysis by Ball et al. [6] has been used in our previous publications [10-12] for evolving our data from the measured $Q^{2}$ to a fixed $Q^{2}=Q_{0}^{2}$. In this paper we shall call 
TABLE I. Comparison of results for the fitted parameters obtained with the two programs. The results are given for fits with an initial scale $Q_{i}^{2}=1 \mathrm{GeV}^{2}$ and $Q_{i}^{2}=10 \mathrm{GeV}^{2}$. All fits are performed in the $\overline{\mathrm{MS}}$ scheme. The uncertainties are statistical only.

\begin{tabular}{ccccc}
\hline \hline & \multicolumn{2}{c}{$Q_{i}^{2}=1 \mathrm{GeV}^{2}$} & \multicolumn{2}{c}{$Q_{i}^{2}=10 \mathrm{GeV}^{2}$} \\
Parameter & Program 1 & Program 2 & Program 1 & \multicolumn{1}{c}{ Program 2 } \\
\hline$\eta_{S}$ & $0.19_{-0.05}^{+0.04}$ & $0.18_{-0.05}^{+0.04}$ & $0.18_{-0.07}^{+0.04}$ & $0.12_{-0.17}^{+0.08}$ \\
$\alpha_{S}$ & $-0.46_{-0.11}^{+0.12}$ & $-0.43_{-0.13}^{+0.13}$ & $-0.61_{-0.13}^{+0.12}$ & $-0.72_{-0.10}^{+0.10}$ \\
$\beta_{S}$ & $3.05_{-0.35}^{+0.38}$ & $3.23_{-0.38}^{+0.41}$ & $3.81_{-0.42}^{+0.43}$ & $3.60_{-0.43}^{+0.63}$ \\
$a_{S}$ & $-13.0_{-1.4}^{+1.2}$ & $-12.2_{-1.5}^{+1.3}$ & $-21.0_{-4.0}^{+2.9}$ & $-22.9_{-6.8}^{+5.2}$ \\
$\eta_{g}$ & $0.21_{-0.21}^{+0.27}$ & $0.38_{-0.28}^{+0.29}$ & $0.22_{-0.18}^{+0.19}$ & $0.61_{-0.55}^{+1.80}$ \\
$\alpha_{g}$ & $0.48_{-1.36}^{+3.36}$ & $1.02_{-1.25}^{+1.44}$ & $0.56_{-0.94}^{+0.95}$ & $-0.44_{-0.48}^{+1.30}$ \\
$\alpha_{\mathrm{NS}}$ & $-0.11_{-0.05}^{+0.05}$ & $-0.12_{-0.05}^{+0.05}$ & $-0.29_{-0.03}^{+0.03}$ & $-0.29_{-0.03}^{+0.03}$ \\
$\beta_{\mathrm{NS}}$ & $1.69_{-0.16}^{+0.16}$ & $1.68_{-0.15}^{+0.15}$ & $2.22_{-0.15}^{+0.16}$ & $2.12_{-0.15}^{+0.16}$ \\
$\chi^{2}$ & 127.4 & 119.8 & 122.6 & 118.8 \\
$N_{\mathrm{DF}}$ & $133-8$ & $133-8$ & $133-8$ & $133-8$ \\
\hline \hline
\end{tabular}

this program 1. Another program for the $Q^{2}$ evolution was developed within the SMC [31]. In this paper we shall call this program 2. This section comments briefly on the evolution programs 1 and 2 . In the next section we present a comparison of results obtained with the two programs in the $\overline{\mathrm{MS}}$ scheme. The comparison of results for polarized parton distributions from two different programs allows us to study the reliability and stability of our results.

In program 1 the Mellin transformation of the evolution equation and the coefficient and splitting functions is used. The DGLAP equations are solved in the moment space with the boundary condition of Eq. (10) at an initial scale value of $Q_{i}^{2}$. The inverse Mellin transformation needed to return to $\left(x, Q^{2}\right)$ space is performed numerically. This is CPU intensive and the computation time goes approximately linearly with the number of data points used in the QCD analysis. For further details on this analysis the reader is referred to Ref. [6].

The other evolution program [31] computes the evolution in $\left(x, Q^{2}\right)$ variables on a grid covering the range of the experimental data. Differentials in $Q^{2}$ are approximated by finite differences. The convolution integrals which appear in Eqs. (4) -(8) are evaluated using the exact form of the splitting and coefficient functions and values for the distribution functions interpolated between adjacent grid points. The convolution integrals of a splitting or coefficient function and a general parton distribution then only need to be computed at the initialization stage of the procedure. In addition, because the parton distributions are evaluated numerically, the method imposes no practical restrictions on their functional forms. The computation time rises roughly linearly with the number of nodes along the $Q^{2}$ axis and roughly as the square of the number of nodes along the $x$ axis. This approximation of the convolution integrals produces satisfactory results if only 30 nodes are used in $x$, which leads to a reduction in computation time of more than two orders of magnitude compared to a straightforward numerical integration. The $Q^{2}$ region of interest was divided into 100 steps. As a check of the accuracy of the method, the numbers of $x$ and $Q^{2}$ points were varied from 30 to 80 and from 100 to 200 , respectively, without producing any significant change in the results.

Result of the comparison. Figure 1 shows the best fits to the $g_{1}^{p, d, n}$ data at the measured $Q^{2}$ obtained using the two programs in the $\overline{\mathrm{MS}}$ factorization scheme and starting the evolution from $Q_{i}^{2}=1 \mathrm{GeV}^{2}$. Since the data do not constrain the high $x$ coefficient $\beta_{g}$ for the gluon, it was fixed to 4.0 from QCD sum rules [30] for all analyses in this paper. The coefficients $a_{f}$ [see Eq. (10)] for the gluon and nonsinglet parton distribution functions were not used in this comparison and we forced the nonsinglet proton and neutron distributions to have the same coefficients $\alpha$ and $\beta$ as was done in Refs. $[6,8] .{ }^{1}$ Both fits describe the data well. The compatibility of the two programs and the invariance with respect to the initial $Q^{2}$ was further tested by repeating the fits with $Q_{i}^{2}$ $=10 \mathrm{GeV}^{2}$. The parameters for the two sets of fits are given in Table I. The quark singlet and nonsinglet coefficients for the parton distributions are nearly the same in both fits and their parameters are consistently (and well) determined by the two programs. On the contrary, the coefficients of the gluon distribution are poorly determined in both programs, and as such the polarized gluon distribution seems to be only marginally determined by the data. Because of the approximate scale independence of $\left(\alpha_{s} \eta_{g}\right)$, since $\alpha_{s}\left(Q^{2}\right)$ reduces by a factor $\sim 2$ between 1 and $10 \mathrm{GeV}^{2}$, the first moment $\eta_{g}$ is expected to increase by the same factor between the two values of $Q^{2}$. The fitted values of $\eta_{g}$ are compatible within their large errors.

The parton distributions obtained in the above fits, performed at $Q_{i}^{2}=1$ and $10 \mathrm{GeV}^{2}$ in the $\overline{\mathrm{MS}}$ scheme, evolved to a fixed $Q_{0}^{2}=5 \mathrm{GeV}^{2}$ are shown in Fig. 2. The singlet and nonsinglet quark distribution functions and their evolution in the two programs are very similar. However, the gluon dis-

\footnotetext{
${ }^{1}$ For the purpose of comparison of the programs such constraints and assumptions make no difference, other than reducing the number of free parameters. Later in this paper when we do fits which are used in the evaluation of integrals we release some of these constraints.
} 


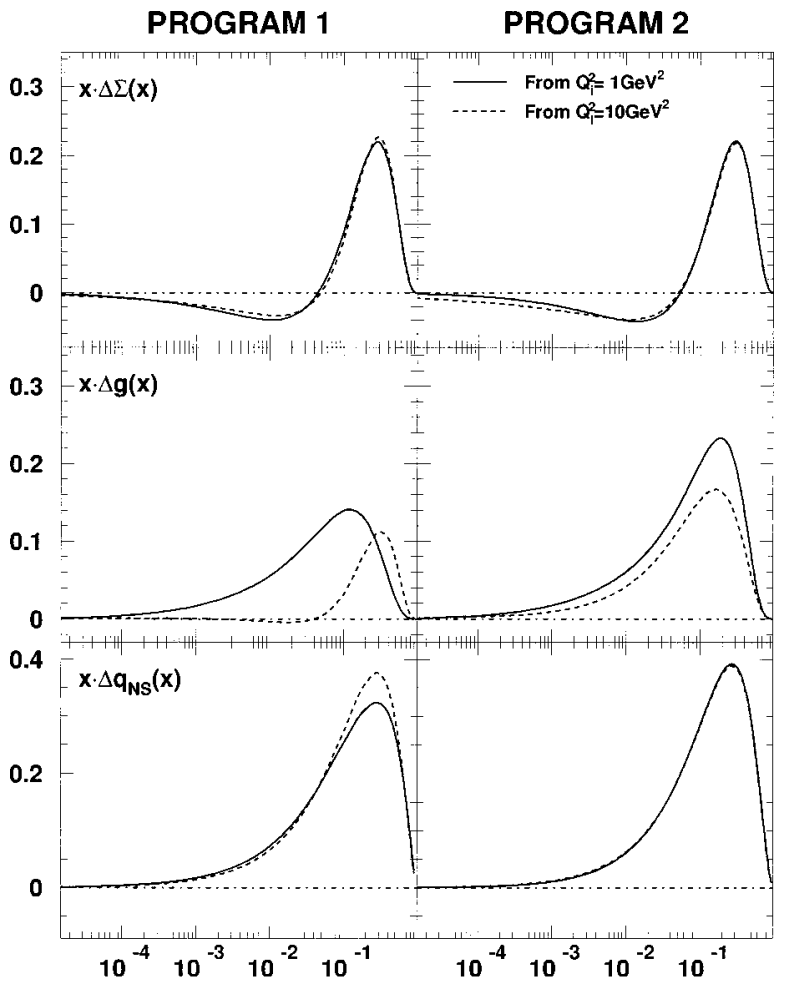

FIG. 2. Polarized parton distribution functions at $Q_{0}^{2}=5 \mathrm{GeV}^{2}$ resulting from the fits using program 1 (left) and program 2 (right) with initial scales of $Q_{i}^{2}=1 \mathrm{GeV}^{2}$ and $10 \mathrm{GeV}^{2}$.

tributions show differences. Keeping in mind the large uncertainty in the determination of gluon distribution coefficients this is not surprising.

Having performed such tests we conclude that given the accuracy of the presently available data different approaches used in the $Q^{2}$ evolution do indeed give consistent results and show similar behaviors as far as the uncertainty esti-

TABLE II. Comparison of results of the QCD fits at $Q^{2}$ $=1 \mathrm{GeV}^{2}$ in the $\overline{\mathrm{MS}}$ scheme and the $\mathrm{AB}$ scheme. The errors are statistical only.

\begin{tabular}{ccc}
\hline \hline Parameter & $\overline{\mathrm{MS}}$ & $\mathrm{AB}$ \\
\hline$\eta_{S}$ & $0.19_{-0.05}^{+0.04}$ & $0.38_{-0.03}^{+0.03}$ \\
$\alpha_{S}$ & $-0.48_{-0.10}^{+0.11}$ & $1.20_{-0.27}^{+0.29}$ \\
$\beta_{S}$ & $3.29_{-0.37}^{+0.40}$ & $4.08_{-0.58}^{+0.63}$ \\
$a_{S}$ & $-13.8_{-1.5}^{+1.3}$ & $(0.0)$ \\
$\eta_{g}$ & $0.25_{-0.22}^{+0.29}$ & $0.99_{-0.31}^{+1.17}$ \\
$\alpha_{g}$ & $0.33_{-1.05}^{+2.05}$ & $-0.70_{-0.20}^{+0.23}$ \\
$\alpha_{\mathrm{NS}}^{p}$ & $-0.19_{-0.08}^{+0.09}$ & $-0.15_{-0.08}^{+0.09}$ \\
$\beta_{\mathrm{NS}}^{p}$ & $1.35_{-0.21}^{+0.23}$ & $1.42_{-0.22}^{+0.23}$ \\
$\alpha_{\mathrm{NS}}^{n}$ & $0.06_{-0.13}^{+0.14}$ & $0.01_{-0.12}^{+0.13}$ \\
$\beta_{\mathrm{NS}}^{n}$ & $2.59_{-0.48}^{+0.52}$ & $2.48_{-0.46}^{+0.51}$ \\
$\chi^{2}$ & 122.9 & 126.3 \\
$N_{\mathrm{DF}}$ & $133-10$ & $133-9$ \\
\hline \hline
\end{tabular}

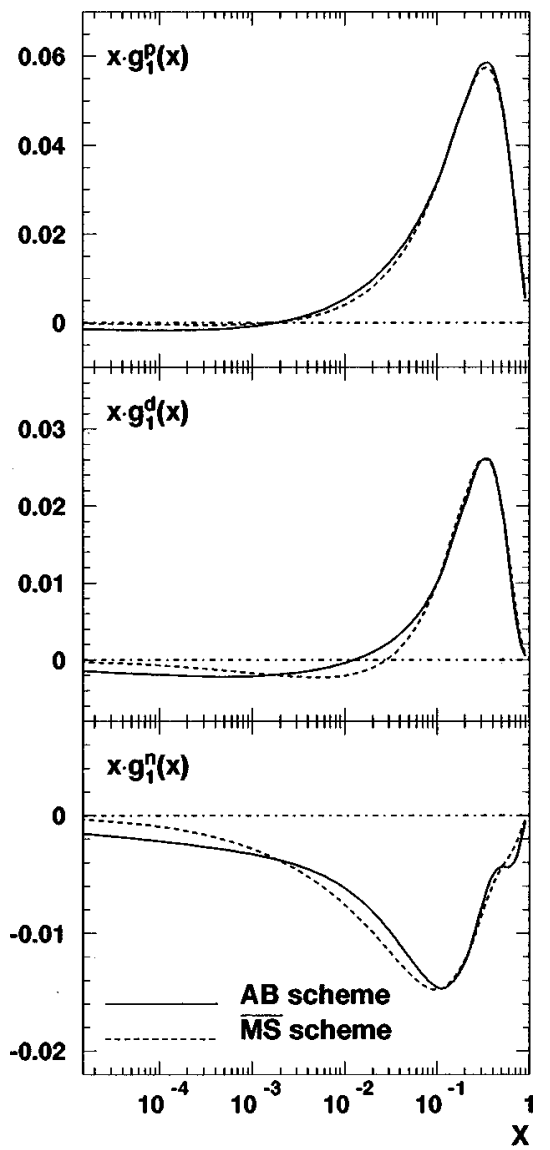

FIG. 3. $x g_{1}^{p, d, n}$ vs $x$. Comparison of fits done in two different schemes $\overline{\mathrm{MS}}$ and $\mathrm{AB}$. All distributions are given at $Q^{2}=5 \mathrm{GeV}^{2}$. The wiggle in $x g_{1}^{n}$ is in a region of $x$ which has little data. The uncertainty in the QCD fit in this region is large (see Fig. 8), consequently, the wiggle itself has no physical significance.

mates are concerned. As mentioned before, an independent paper on the QCD analysis in program 1 has been published [6]. This program has been used previously in the analysis of SMC data [10-12] and required minimal modification to study the evolution in the two factorization schemes [AdlerBardeen $(\mathrm{AB})$ and $\overline{\mathrm{MS}}]$. In order to preserve continuity with our previous publications and in view of the fact that programs 1 and 2 provide consistent results, from now on we will present results using program 1 exclusively.

\section{E. Comparison of results in $\overline{\mathrm{MS}}$ and $\mathrm{AB}$ schemes}

The values of the fitted parameters obtained in the $\overline{\mathrm{MS}}$ and $\mathrm{AB}$ schemes for the initial $Q_{i}^{2}=1 \mathrm{GeV}^{2}$ are listed in Table II. In this comparison we have released the constraint requiring the shape of the nonsinglet parton distribution in the proton and neutron to be the same, i.e., we allow different values of $\alpha, \beta$ in the $\Delta q_{\mathrm{NS}}$ of the proton and neutron. The nearly equal values of the $\chi^{2}$ show that the data are equally well described by the analyses performed in the two schemes with the input parametrizations of Eq. (10). In other words, the functional form of the initial parton distributions in Eq. (10) is flexible enough to describe the data. We observe in 


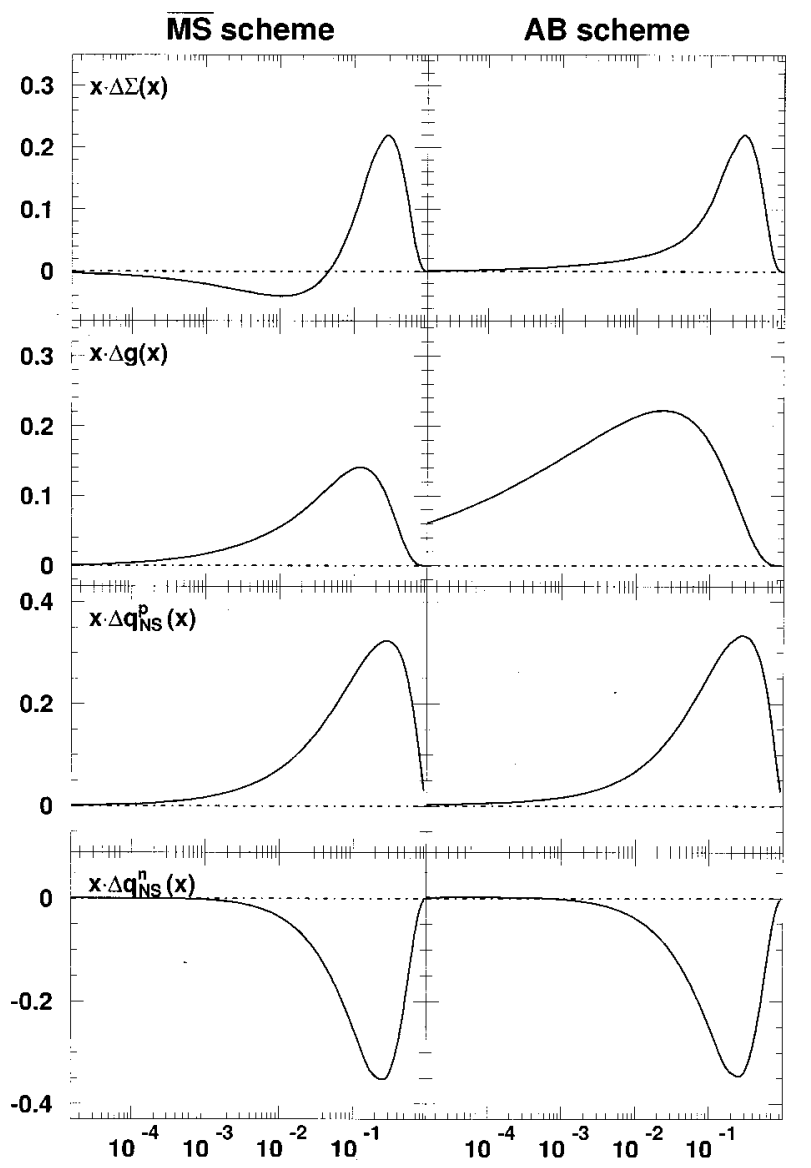

FIG. 4. Polarized parton distribution functions at $Q_{0}^{2}=5 \mathrm{GeV}^{2}$ obtained in two different schemes, $\overline{\mathrm{MS}}$ and $\mathrm{AB}$.

Fig. 3 that the fitted $g_{1}(x)$ distributions, evolved to the reference $Q_{0}^{2}=5 \mathrm{GeV}^{2}$, differ very little in the range $0.003<x$ $<0.8$ in which spin structure function data are available.

The comparison of the fitted polarized parton distributions (Fig. 4) clearly shows how the two schemes differ in the singlet sector. In the $\overline{\mathrm{MS}}$ scheme $\Delta \Sigma$ is constrained by the negative values of $g_{1}^{d}(x)$ at low $x$ to become negative for $x$ $\leq 0.05$. The crossover $x_{0}$ is determined by the linear term in $x\left(a_{s}=-1 / x_{0}\right)$. In the $\mathrm{AB}$ scheme, this term is not needed because $\Delta \Sigma$ remains positive over the full range of the data. The polarized gluon distribution is found to be larger in the $\mathrm{AB}$ scheme and is shifted to lower values of $x$ compared to that in the $\overline{\mathrm{MS}}$ scheme. Differences of the same order between gluon determinations in the two schemes have been reported in a previous analysis [9] by the E154 Collaboration. Within the precision of the data, the first moments of the polarized singlet and gluon distributions obtained in the two schemes are compatible with the relation in Eq. (9) at the $Q^{2}$ value of $1 \mathrm{GeV}^{2}$.

The principal aim of the experimental collaborations is the measurement of the first moments of spin structure functions $g_{1}^{p, d, n}$. Since the analyses done in both schemes seem to describe the $g_{1}$ data equally well it does not matter which scheme we follow. In the past we have used the AB scheme for our results [10-12]. In order to keep continuity with those publications we use the $\mathrm{AB}$ factorization scheme in
TABLE III. The best fit parameters of the PQCD fit when $\alpha_{s}\left(M_{Z}^{2}\right)$ was made a free parameter. All parameters are given at $Q^{2}=1 \mathrm{GeV}^{2}$ except for the value of $\alpha_{s}$ which is given at $Q^{2}$ $=M_{Z}^{2} \mathrm{GeV}^{2}$. The uncertainties are statistical only.

\begin{tabular}{cccc}
\hline \hline Parameter & Value & Parameter & Value \\
\hline$\eta_{S}$ & $0.39_{-0.03}^{+0.03}$ & $\eta_{g}$ & $0.98_{-0.37}^{+7.41}$ \\
$\alpha_{S}$ & $1.22_{-0.27}^{+0.28}$ & $\alpha_{g}$ & $-0.78_{-0.21}^{+0.22}$ \\
$\beta_{S}$ & $4.00_{-0.60}^{+0.63}$ & $\beta_{g}$ & $(4.0)$ \\
$\eta_{\mathrm{NS}}^{p}$ & $\frac{3}{3}\left|\frac{g_{A}}{g_{V}}\right|+\frac{1}{4} a_{8}$ & $\eta_{\mathrm{NS}}^{n}$ & $-\frac{3}{4}\left|\frac{g_{A}}{g_{V}}\right|+\frac{1}{4} a_{8}$ \\
$\alpha_{\mathrm{NS}}^{p}$ & $-0.08_{-0.10}^{+0.11}$ & $\alpha_{\mathrm{NS}}^{n}$ & $0.04_{-0.13}^{+0.14}$ \\
$\beta_{\mathrm{NS}}^{p}$ & $1.53_{-0.24}^{+0.26}$ & $\beta_{\mathrm{NS}}^{n}$ & $2.60_{-0.49}^{+0.54}$ \\
$\alpha_{s}\left(M_{Z}^{2}\right)$ & \multicolumn{3}{c}{$0.121_{-0.002}^{+0.002}$} \\
$\chi^{2}$ & \multicolumn{3}{c}{125.1} \\
$N_{\mathrm{DF}}$ & \multicolumn{3}{c}{$133-10$} \\
\hline \hline
\end{tabular}

this paper for all further analysis. We will call this the standard fit.

\section{F. Comments on the determination of $\alpha_{s}\left(Q^{2}\right)$}

The analysis presented so far starts with the spindependent virtual photon-nucleon asymmetries measured by different experiments. We determine from these asymmetries the spin-dependent structure functions $g_{1}$ using parametrizations of the unpolarized structure functions $F_{2}$ and $R$. The information on scaling violations from the unpolarized nucleon structure functions $F_{2}$ (which are measured with significantly better accuracy compared to $g_{1}$ ), is hence an input to the analysis. These scaling violations have been studied and have led to a determination of the strong coupling constant $\alpha_{s}$ [32].

In a recent publication [8] it was shown that the value of $\alpha_{s}$ can be extracted using PQCD analysis of the spin structure function data, while results from another analysis [9] indicated that their analysis was sensitive to the value of $\alpha_{s}\left(M_{Z}^{2}\right)$ used.

To check the sensitivity of our analysis to the value of $\alpha_{s}$, we make the value of $\alpha_{s}\left(M_{Z}^{2}\right)$ (which normally is an input parameter in the PQCD analysis) a free parameter in the fit. Table III shows the fitted values and the statistical uncertainties in the parameters at $Q_{i}^{2}=1 \mathrm{GeV}^{2}$. The values change little in comparison with those presented before in Table II for the $\mathrm{AB}$ scheme. Estimation of uncertainties due to experimental systematic effects in the data and those of theoretical origins (procedure will be described in Sec. II G) gives

$$
\alpha_{s}\left(M_{Z}^{2}\right)=0.121 \pm 0.002(\text { stat }) \pm 0.006 \text { (syst and theory). }
$$

The value of $\alpha_{s}\left(M_{Z}^{2}\right)$ indeed comes out to be consistent with that determined from the PQCD analyses of the unpolarized data. As such, while the determination of $\alpha_{s}$ is certainly possible using the scaling violations of $g_{1}$, with the presently available data on $A_{1}$ it is difficult to separate the information on scaling violations due to $F_{2}$ and due to $A_{1}$. In this 

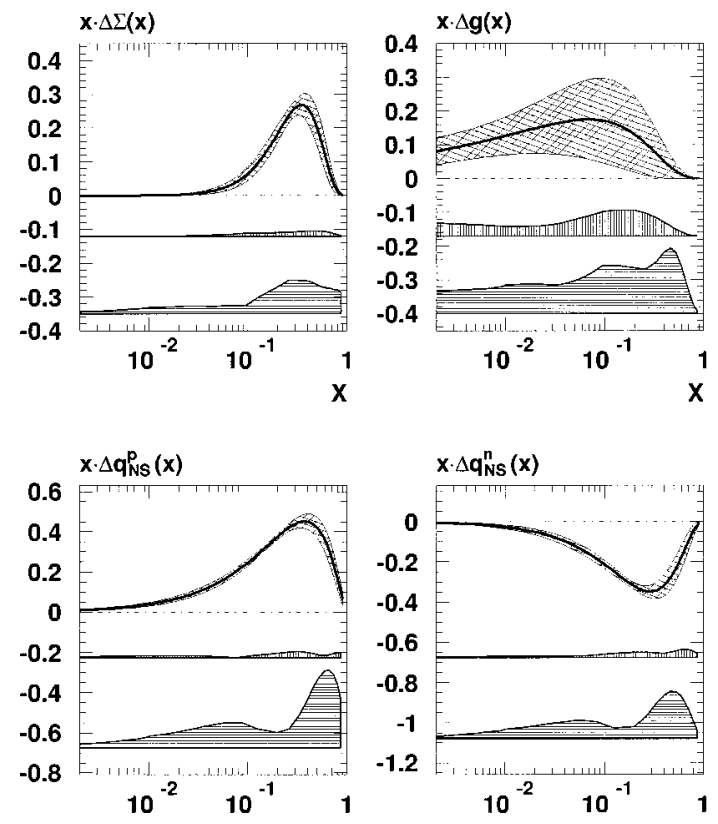

FIG. 5. Polarized parton distribution functions determined from the PQCD analysis at $Q_{i}^{2}=1 \mathrm{GeV}^{2}$. Their statistical uncertainty as obtained from the QCD fit is shown by a band with crossed hatch. The experimental systematic uncertainty is indicated by the vertically hatched band, and the theoretical uncertainty by the horizontally hatched band.

paper we henceforth always take the value of the strong coupling constant $\alpha_{s}\left(M_{Z}^{2}\right)=0.118 \pm 0.003$ as given in Ref. [28].

\section{G. Evaluation of uncertainties in the polarized parton distribution functions}

Figure 5 shows the results for the parton distributions and their uncertainties. In the calculation of the $\chi^{2}$ [Eq. (11)] only the statistical uncertainty on the data points was used. The uncertainty in the parton distribution due to this is shown (cross hatch) with the parton distribution (bold line in the cross hatch).

To estimate the uncertainty in a parton distribution function due to the experimental systematic errors the following procedure was used. For each data set the experimental systematic uncertainties on $A_{1}$ due to all sources ( $\sigma_{\text {syst }}^{i}$ ) were added in quadrature to calculate a total systematic uncertainty $\left(\sigma_{\text {syst }}^{T}\right)$ for that data set. The QCD fits were then repeated with input values of asymmetries $A_{1} \pm \sigma_{\text {syst }}^{T}$. The unpolarized structure function $F_{2}$ and $R$ used to evaluate $g_{1}$ from $A_{1}$ were shifted to the upper and lower limits of their respective parametrizations to estimate their contribution to the uncertainty. Then these experimental, $F_{2}$, and $R$ contributions were added quadratically. The resulting envelopes of uncertainty are shown in Fig. 5 (vertically hatched band) as a function of $x$.

In addition to the statistical and systematic uncertainties a significant source of uncertainty in the parton distribution functions comes from uncertainty in the various input parameters to the PQCD analysis. We call them "theoretical" uncertainties. They include uncertainties in the values of fac-
TABLE IV. First moments of the nucleon spin structure functions at $Q_{0}^{2}=5 \mathrm{GeV}^{2}$ in the measured $x$ range from 0.003 to 0.8 . The first uncertainty is statistical, the second experimental systematic, and the third due to the uncertainty in evolution. For comparison, the integral over the QCD fit is given in the third column.

\begin{tabular}{lcc}
\hline \hline Nucleon & $\int_{0.003}^{0.8} g_{1}\left(x, Q_{0}^{2}\right) d x$ & $\int_{0.003}^{0.8} g_{1}^{\mathrm{fit}}\left(x, Q_{0}^{2}\right) d x$ \\
\hline Proton & $0.130 \pm 0.003 \pm 0.005 \pm 0.004$ & 0.132 \\
Deuteron & $0.036 \pm 0.004 \pm 0.003 \pm 0.002$ & 0.040 \\
Neutron & $-0.054 \pm 0.007 \pm 0.005 \pm 0.004$ & -0.048 \\
\hline \hline
\end{tabular}

torization and renormalization scales, the value of $\alpha_{s}$, the functional form of the initial parton distribution function, the values of quark mass thresholds, and the value of $g_{A} / g_{V}$. We evaluated them by varying each of these parameters by their known errors (whenever available). The uncertainties in the factorization and renormalization scales are related to the uncertainty in the result due to the neglect of higher order corrections in the PQCD analysis. This was estimated by independently varying factorization and renormalization scale factors $k_{1}$ and $k_{2}$ in Sec. II C by 2 in both directions, i.e., $0.5 \leqslant k_{1}, k_{2} \leqslant 2.0$. For the standard fit the value of $\alpha_{s}\left(M_{Z}^{2}\right)=0.118$ was used. This value was varied between $0.118 \pm 0.003$. Another input to our analysis is the assumed functional form of Eq. (10), the initial parton distribution function. To evaluate its effect on the results two tests were done. First, we used different combinations of constraints on the parameters $\alpha_{f}, \underline{\beta}_{f}$, and $a_{f}$ in Eq. (10) including also an additional term $b \sqrt{x}$ in the polynomial. If the confidence level of the resulting fit was comparable to that of the best fit, then that functional form was accepted and the result of the fit was considered for estimating the uncertainty due to the functional form of the initial parton distribution. Second, we started at an initial scale $Q_{i}^{2}$ different from $1 \mathrm{GeV}^{2}$ and observed how different the resultant parton distributions were when evolved to the same common $Q_{0}^{2}$. The theoretical systematic uncertainty bands were then added in quadrature (as functions of $x$ ). The envelopes of such uncertainty as a function of $x$ for singlet and nonsinglet parton distributions are shown in Fig. 5 by the horizontally hatched bands. The dominant uncertainties were due to the uncertainty in the factorization scale $M^{2}$, the renormalization scale $\mu^{2}$, and due to the uncertainty in the assumed functional form of the initial parton distributions.

\section{QCD ANALYSIS-RESULTS}

\section{A. Evaluation of first moments at fixed $Q_{0}^{2}$}

We use all available data in the kinematic region $Q^{2}$ $\geqslant 1 \mathrm{GeV}^{2}, x \geqslant 0.003$ to evaluate $\Gamma_{1}=\int_{0}^{1} g_{1}(x) d x$ at a fixed $Q^{2}$. Starting from $g_{1}\left(x, Q^{2}\right)$ at the measured $x$ and $Q^{2}$ we obtain $g_{1}$ at a fixed $Q_{0}^{2}$ as follows:

$$
g_{1}\left(x, Q_{0}^{2}\right)=g_{1}\left(x, Q^{2}\right)+\left[g_{1}^{\mathrm{fit}}\left(x, Q_{0}^{2}\right)-g_{1}^{\mathrm{fit}}\left(x, Q^{2}\right)\right],
$$

where $g_{1}^{\text {fit }}\left(x, Q_{0}^{2}\right)$ and $g_{1}^{\text {fit }}\left(x, Q^{2}\right)$ are the values of $g_{1}$ evaluated at $Q_{0}^{2}$ and $Q^{2}$ of the experiment using the fit parameters, 
TABLE V. First moments of the structure functions at $Q_{0}^{2}$ $=5 \mathrm{GeV}^{2}$ from the unmeasured $x$ regions and their total uncertainties due to the experimental systematics and the theoretical sources in the evolution.

\begin{tabular}{ccc}
\hline \hline $\int g_{1}^{\text {fit }}\left(x, Q_{0}^{2}\right) d x$ & $0.0<x<0.003$ & $0.8<x<1.0$ \\
\hline Proton & $-0.012_{-0.025}^{+0.014}$ & $0.003_{-0.001}^{+0.001}$ \\
Deuteron & $-0.015_{-0.023}^{+0.010}$ & $0.000_{-0.001}^{+0.000}$ \\
Neutron & $-0.020_{-0.026}^{+0.010}$ & $0.000_{-0.001}^{+0.001}$ \\
\hline \hline
\end{tabular}

respectively. ${ }^{2}$ We choose $Q_{0}^{2}=5 \mathrm{GeV}^{2}$ which is close to the average $Q^{2}$ of the world data set used in the analysis. In the measured range $0.003<x<0.8$ the contributions to the first moments of the nucleon structure functions calculated from the data are given in Table IV, column 2. The first uncertainty is statistical, the second is systematic and the third is due to the uncertainty in the $Q^{2}$ evolution. The method used for combining different data sets is discussed in Refs. [1, 33, 34]. Figures 6, 7, 8 and their insets show $x g_{1}^{p, n, d}$, respectively, as a function of $x$. The areas under the $g_{1}^{\text {fit }}$ curves are given in Table IV, column 3. The integrals calculated in both ways are very similar.

To estimate the contributions to the first moment from the unmeasured low $x(x<0.003)$ and high $x(x>0.8)$ regions, we integrate over $g_{1}^{\text {fit }}$ calculated at $Q^{2}=5 \mathrm{GeV}^{2}$ using the parameters for the parton distributions. The central values and the uncertainties in the low and high $x$ contributions are given in Table $\mathrm{V}$. The areas under the QCD fit for $x<0.003$ in Figs. 6, 7, and 8 and their insets correspond to the low $x$ contribution. The uncertainties in the low and high $x$ integrals are obtained using the same procedure as for the estimation of the uncertainty in the QCD evolution described in Sec. II G. Had we taken the traditional approach [10-12] of using Regge extrapolation in the low $x$ region and a constant $A_{1}$ in the high $x$ unmeasured region (bounded by $A_{1}$ $<1$ ), we would get results using the present data consistent with those presented in Table $\mathrm{V}$, but with significantly smaller uncertainties (see Ref. [11] for a detailed discussion).

The low $x$ contributions to the first moments quoted in Table $\mathrm{V}$ rely on the validity of the assumption that the parton distribution functions behave as $x^{\alpha}$ at the initial $Q^{2}=Q_{i}^{2}$ when $x \rightarrow 0$ with the values of $\alpha$ quoted in Table II for the AB scheme. Under this condition $g_{1}^{P}\left(x, Q_{0}^{2}\right)$ becomes negative below $x \simeq 0.001$, i.e., slightly below the lowest $x$ data available (Fig. 6). The $g_{1}^{d}\left(x, Q_{0}^{2}\right)$ becomes negative below $x$ $=0.02$ (Fig. 7), while $g_{1}^{n}\left(x, Q_{0}^{2}\right)$ is negative for all $x$ (Fig. 8). Other functional behaviors of $g_{1}$ at low $x(x<0.003)$ have been investigated. The resulting contributions to the moments were found to be in the range of systematic errors quoted in Table $\mathrm{V}$.

The uncertainties in $\Gamma_{1}^{p, d, n}\left(Q_{0}^{2}\right)$, for $Q_{0}^{2}=5 \mathrm{GeV}^{2}$ are separated by sources in Table VI. The experiments giving

\footnotetext{
${ }^{2}$ From now on the superscript " fit", indicates that the quantity was calculated using the best fit parameters of the QCD fit.
}

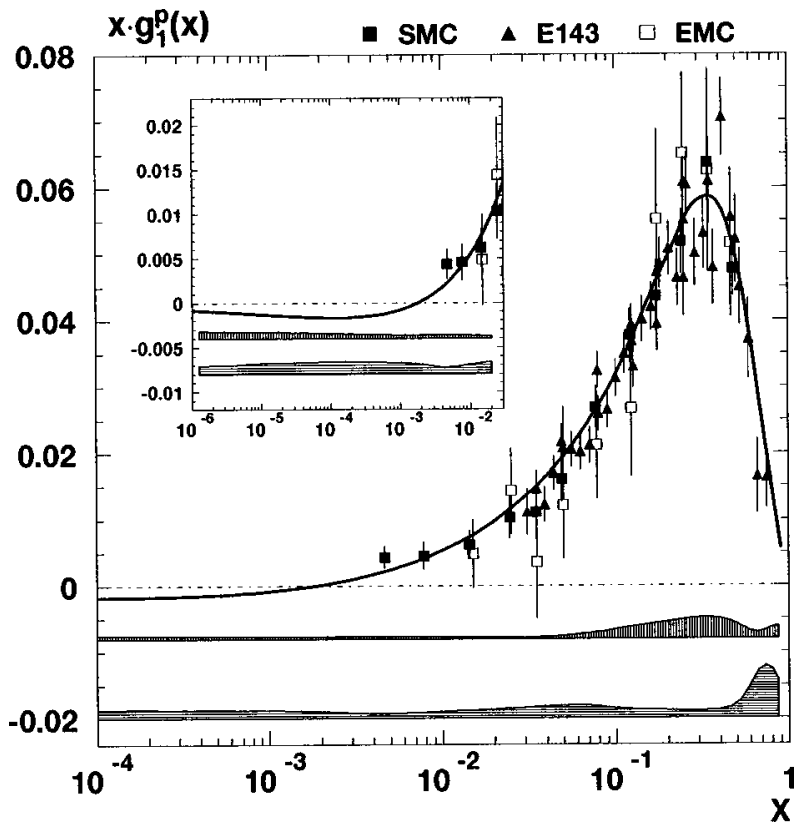

FIG. 6. $x g_{1}^{p}$ vs $x$ for the world data with the QCD fit at $Q^{2}$ $=5 \mathrm{GeV}^{2}$. The low $x$ region is emphasized in the inset. The data points are shown with their statistical errors. The uncertainties of the fit due to experimental systematics and theoretical sources are shown by the vertically and horizontally hatched bands, respectively.

the largest three contributions are listed and the remaining ones are added together in "other exp." The largest three theoretical sources of errors, namely, the factorization and renormalization scales, the value of $\alpha_{s}$, and the uncertainty in the form of initial parton distribution functions are also given separately. The rest of the sources such as the uncertainties in the quark mass thresholds, the values of the constants $g_{A} / g_{V}, a_{8}$, etc., are collected as one source and called "others."

Our best estimate for the first moments $\Gamma_{1}^{p, d, n}\left(Q_{0}^{2}\right.$ $=5 \mathrm{GeV}^{2}$ ) over the full $x$ range is given in the second column of Table VII. The first uncertainty is statistical and the second is systematic. The third uncertainty is due to the low and high $x$ extrapolation and the $Q^{2}$ evolution; they are correlated and are both of theoretical origin. The third column of this table gives the values of the first moments at $Q_{0}^{2}$ $=10 \mathrm{GeV}^{2}$ using the SMC data in the measured $x$ range.

\section{B. $\Delta g$ and $a_{0}$ determination}

\section{1. $\Delta g\left(Q_{0}^{2}\right)$ and its evolution}

Our analysis performed in the $\mathrm{AB}$ scheme using an initial $Q_{i}^{2}=1 \mathrm{GeV}^{2}$ results in

$$
\begin{aligned}
\eta_{g} & =\int_{0}^{1} \Delta g\left(Q^{2}=1 \mathrm{GeV}^{2}\right) d x \\
& =0.99_{-0.31}^{+1.17}(\mathrm{stat})_{-0.22}^{+0.42}(\mathrm{syst})_{-0.45}^{+1.43}(\mathrm{th}) .
\end{aligned}
$$

The procedure used to estimate the uncertainties was the same as described in Sec. II G. When evolved to 5 and 10 $\mathrm{GeV}^{2}$ the values of $\eta_{g}$ become 1.7 and 2.0 , respectively. The 


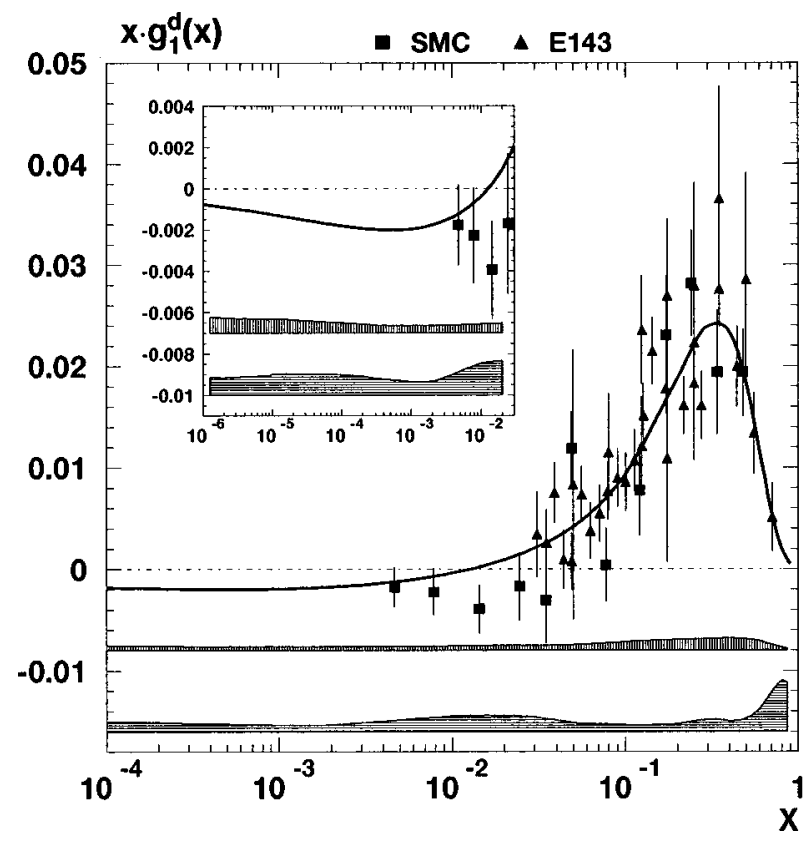

FIG. 7. $x g_{1}^{d}$ vs $x$ for the world data with the QCD fit at $Q^{2}$ $=5 \mathrm{GeV}^{2}$. The low $x$ region is emphasized in the inset. The data points are shown with their statistical errors. The uncertainties of the fit due to experimental systematics and theoretical sources are shown by the vertically and horizontally hatched bands, respectively.

analysis indicates that the uncertainty in the measurement of this quantity is large. Very little can be said about this quantity on the basis of the present data. Measurements in which the gluon is involved in the leading order (such as the photon-gluon fusion process) are needed, in addition to more precise DIS data on $g_{1}$, for an improved determination of $\eta_{g}$.

\section{2. $a_{0}$ determination}

The values of the singlet axial current matrix element $a_{0}$ determined from the fits are shown in Fig. 9 for values of $Q_{i}^{2}=1,4,7,10 \mathrm{GeV}^{2}$ in the $\overline{\mathrm{MS}}$ and $\mathrm{AB}$ schemes. The estimated uncertainty is shown for $Q_{i}^{2}=1 \mathrm{GeV}^{2}$ only. The uncertainties at higher $Q_{i}^{2}$ are comparable. The solid curve is a calculation for the $Q^{2}$ dependence of $a_{0}$ based on the best fit performed with $Q_{i}^{2}=1 \mathrm{GeV}^{2}$ in $\overline{\mathrm{MS}}$ scheme. The results obtained in this scheme for higher $Q_{i}^{2}$ values fall consistently on this curve. For $Q^{2}>1 \mathrm{GeV}^{2}$ the $Q^{2}$ dependence is weak and is below the sensitivity of the existing data. In the $\overline{\mathrm{MS}}$ scheme, $a_{0}$ is identified with the integral $\eta_{S}$ of the singlet quark distribution (Table II) while in the $\mathrm{AB}$ scheme the gluon contribution must be subtracted:

$$
a_{0}\left(Q^{2}\right)=\eta_{S}^{\mathrm{AB}}-n_{f} \frac{\alpha_{s}\left(Q^{2}\right)}{2 \pi} \eta_{g}\left(Q^{2}\right) .
$$

Figure 9 shows that the world data are good enough to test the above relation. In the $\mathrm{AB}$ scheme at $Q_{0}^{2}=1 \mathrm{GeV}^{2}$ we get $a_{0}=0.23 \pm 0.07$ (stat) \pm 0.19 (syst) while at the same $Q_{0}^{2}$ in the $\overline{\mathrm{MS}}$ scheme we get $a_{0}=0.19 \pm 0.05$ (stat) \pm 0.04 (syst). These

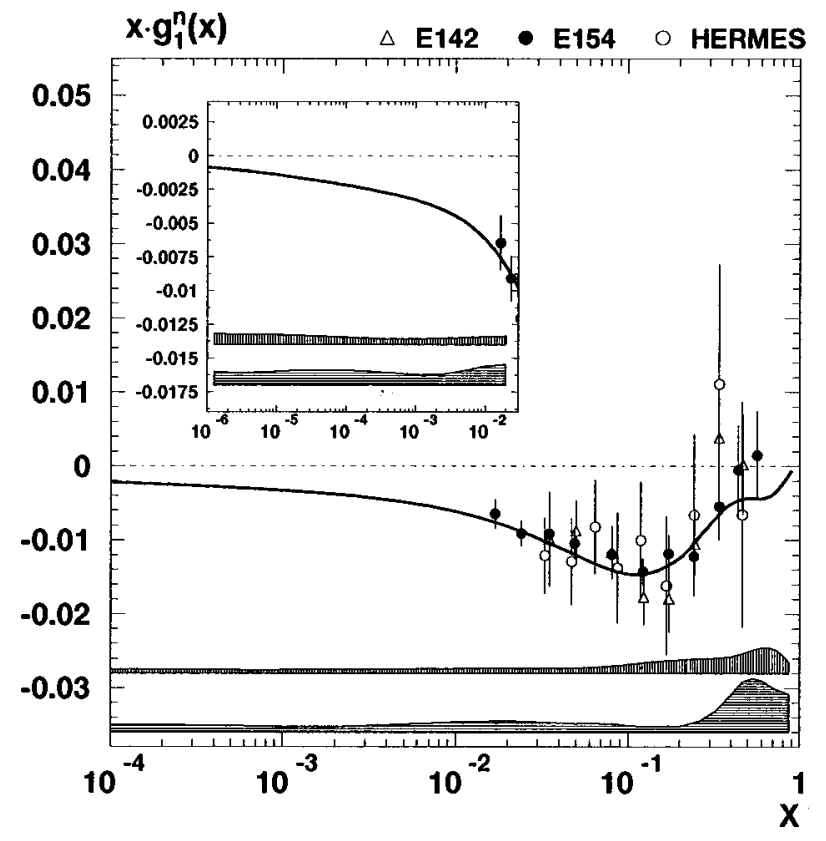

FIG. 8. $x g_{1}^{n}$ vs $x$ for the world data with the QCD fit at $Q^{2}$ $=5 \mathrm{GeV}^{2}$. Only statistical errors are shown with the data points. The low $x$ region is emphasized in the inset. The uncertainties in the fit due to experimental systematics and theoretical sources are shown by the vertically and horizontally hatched bands, respectively.

values are compatible within errors as required for a scheme independent quantity and correspond to about $\frac{1}{3}$ of the naive quark-parton model (QPM) expectation $a_{0}=a_{8} \simeq 0.58$. The

TABLE VI. Uncertainties on the first moments resulting from the PQCD analysis separated by sources given in this table in three parts. In the top part the first moments of $g_{1}^{p, d, n}$ at $Q_{0}^{2}=5 \mathrm{GeV}^{2}$ are given with their total experimental systematic and theoretical uncertainties. In the central part the total experimental systematic uncertainty from above is split into contributions from different experiments, while in the lowest part the total theoretical uncertainty is split into its sources.

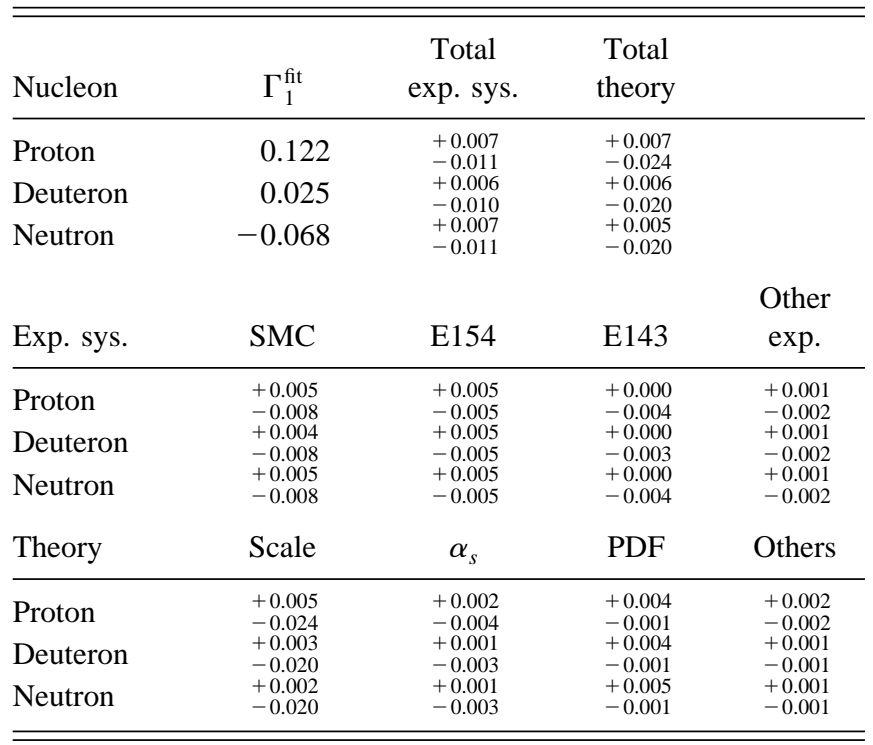


TABLE VII. Table of $\Gamma_{1}^{p, d, n}$ at $Q_{0}^{2}=5 \mathrm{GeV}^{2}$ for the world set of data (left), and at $Q_{0}^{2}=10 \mathrm{GeV}^{2}$ for SMC (right).

\begin{tabular}{lrr}
\hline \hline & $\begin{array}{c}\text { World } \\
\Gamma_{1}\left(Q_{0}^{2}\right)\end{array}$ & \multicolumn{1}{c}{$\begin{array}{c}\text { SMC } \\
Q_{0}^{2}=5 \mathrm{GeV}^{2}\end{array}$} \\
\hline Proton & $0.121 \pm 0.003 \pm 0.005 \pm 0.017$ & \multicolumn{1}{c}{$Q_{0}^{2}=10 \mathrm{GeV}^{2}$} \\
Deuteron & $0.021 \pm 0.004 \pm 0.003 \pm 0.016$ & $0.120 \pm 0.005 \pm 0.006 \pm 0.014$ \\
Neutron & $-0.075 \pm 0.007 \pm 0.005 \pm 0.019$ & $-0.078 \pm 0.013 \pm 0.008 \pm 0.014$ \\
\hline \hline
\end{tabular}

systematic errors in the $a_{0}$ determined from the analysis in the $\mathrm{AB}$ scheme is larger than the one determined in the $\overline{\mathrm{MS}}$ scheme because of the correlation introduced by $\Delta g$ and its uncertainty in the evaluation [see Eq. (15)].

The first moments $\Gamma^{p, n, d}$ can also be expressed in terms of the matrix elements $a_{0}, a_{3}$ and $a_{8}$ [2]. If exact $\mathrm{SU}(3)$ flavor symmetry is assumed for the axial octet current, $a_{3}$ and $a_{8}$ are given by the coupling constants for neutron and hyperon decays $a_{3}=F+D$ and $a_{8}=3 F-D$, respectively. Under this assumption and using the input values quoted in Table VII we obtain at $Q^{2}=5 \mathrm{GeV}^{2} a_{0}=0.13 \pm 0.17$. This result is consistent with those obtained before (directly from QCD analysis) but note that in the measured $x$ range the same $Q^{2}$ evolution has been used in all these results.

It has often been suggested that the difference between the low experimental value of $a_{0}$ and its naive QPM prediction could be explained by a large gluon contribution. The value of $\eta_{S}=0.38_{-0.03-0.02-0.05}^{+0.03+0.03+03}$ in the $\mathrm{AB}$ scheme (only statistical uncertainty on $\eta_{S}$ is shown in Table II), obtained in

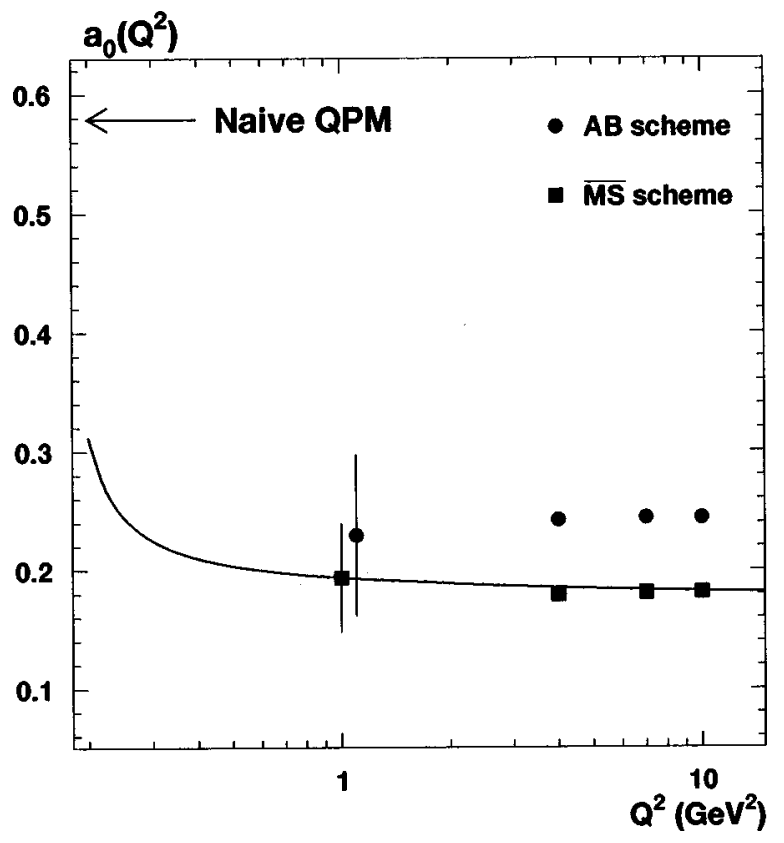

FIG. 9. The $Q^{2}$ dependence of $a_{0}$ determined in this analysis using different schemes and with different starting scales $Q_{i}^{2}$ is shown. The curve shows the predicted $Q^{2}$ evolution of $a_{0}$ in the $\overline{\mathrm{MS}}$ scheme. The statistical errors in $a_{0}$ are shown only for $Q_{i}^{2}$ $=1 \mathrm{GeV}^{2}$. The uncertainties for the other points at higher $Q_{i}^{2}$ values are comparable. The expectation for the value of $a_{0}$ based on the naive QPM is also shown for comparison. this analysis does not support this suggestion.

\section{Determination of Bjorken sum rule}

\section{Bjorken sum rule from $Q C D$ analysis}

The Bjorken sum rule is a fundamental result in PQCD. In this section we present a method of testing this in a way consistent with the PQCD analysis presented so far. The conventional method of testing the Bjorken sum rule (which has been used in most experimental papers) is to evaluate the difference between the first moments of the proton and neutron polarized structure functions at a fixed $Q_{0}^{2}$ and to see if the relation

$$
\Gamma_{1}^{p}-\Gamma_{1}^{n}=\frac{1}{6}\left|\frac{g_{A}}{g_{V}}\right| C_{1}^{\mathrm{NS}}\left(Q^{2}\right)
$$

holds. Here $g_{A} / g_{V}$ is the axial vector coupling constant. The coefficient $C_{1}^{\mathrm{NS}}\left(Q^{2}\right)$ has been calculated to fourth order in $\alpha_{s}\left(Q^{2}\right)$ [35].

Based on the PQCD analysis we have evaluated the first moments of the proton and neutron structure functions at $Q_{0}^{2}=5 \mathrm{GeV}^{2}$ given in Table VII. However, we cannot directly use them to evaluate the Bjorken sum rule because in this analysis we have taken the first moments to be $\eta_{\mathrm{NS}}^{p / n}=$ $\pm \frac{3}{4}\left|g_{A} / g_{V}\right|+\frac{1}{4} a_{8}$, with the value of $g_{A} / g_{V}$ fixed to its nominal value of $1.2601 \pm 0.0025$ [28]. In this way the Bjorken sum rule is assumed in the analysis. We can test the validity of the Bjorken sum rule by releasing this constraint in our PQCD analysis and making $g_{A} / g_{V}$ one of the free parameters to be fitted by the $g_{1}$ data. The best fit parameters for such a fit are given in Table VIII. The experimental and theoretical uncertainty study presented in Sec. II G was repeated for the uncertainty estimation for $g_{A} / g_{V}$. We obtain

$$
\left|\frac{g_{A}}{g_{V}}\right|=1.15_{-0.03}^{+0.03}(\text { stat })_{-0.06}^{+0.07}(\mathrm{syst})_{-0.04}^{+0.14}(\mathrm{th}) .
$$

The value of $g_{A} / g_{V}$ determined here is consistent with the nominal value used above. The uncertainties (particularly theoretical) are large. The largest contribution to the theoretical uncertainty is the factorization and renormalization scales and due to the choice of the initial parton distributions.

The above value of $g_{A} / g_{V}$ and its uncertainty when used to evaluate the value of Bjorken sum in Eq. (16) to order $\mathcal{O}\left(\alpha_{s}\right)$ (consistent with all other analysis presented in this paper) at $Q_{0}^{2}=5 \mathrm{GeV}^{2}$ gives 
TABLE VIII. Best parameters at $Q^{2}=1 \mathrm{GeV}^{2}$ when $g_{A} / g_{V}$ is a free parameter in the fit. The uncertainties shown are statistical only.

\begin{tabular}{cccc}
\hline \hline Parameter & Value & Parameter & Value \\
\hline$\eta_{S}$ & $0.38_{-0.02}^{+0.03}$ & $\eta_{g}$ & $0.94_{-0.29}^{+1.26}$ \\
$\alpha_{S}$ & $1.03_{-0.27}^{+0.29}$ & $\alpha_{g}$ & $-0.71_{-0.21}^{+0.22}$ \\
$\beta_{S}$ & $3.64_{-0.59}^{+0.63}$ & $\beta_{g}$ & 4.0 \\
$\left|\frac{g_{A}}{g_{V}}\right|$ & & $1.15_{-0.03}^{+0.03}$ & $3\left|\frac{g_{A}}{g_{V}}\right|+\frac{1}{4} a_{8}$ \\
$\eta_{\mathrm{NS}}^{p}$ & $\frac{3}{4}\left|\frac{g_{A}}{g_{V}}\right|+\frac{1}{4} a_{8}$ & $\eta_{\mathrm{NS}}^{n}$ & $0.20_{-0.14}^{+0.16}$ \\
$\alpha_{\mathrm{NS}}^{p}$ & $-0.01_{-0.10}^{+0.10}$ & $\alpha_{\mathrm{NS}}^{n}$ & $3.48_{-0.63}^{+0.70}$ \\
$\beta_{\mathrm{NS}}^{p}$ & $1.86_{-0.28}^{+0.30}$ & $\beta_{\mathrm{NS}}^{n}$ & \\
$\chi^{2}$ & & 116.1 & \\
$N_{\mathrm{DF}}$ & & $133-10$ & \\
\hline
\end{tabular}

$\Gamma_{1}^{p}-\Gamma_{1}^{n}=0.174_{-0.005}^{+0.005}(\mathrm{stat})_{-0.009}^{+0.011}(\mathrm{syst})_{-0.006}^{+0.021}(\mathrm{th})=0.174_{-0.012}^{+0.024}$,

which is in excellent agreement with the theoretically calculated value of $\Gamma_{1}^{p}-\Gamma_{1}^{n}=0.181 \pm 0.003[35]$ at the same $Q_{0}^{2}$.

\section{QCD evolution of $g_{1}^{N S}$}

An alternative way to determine the Bjorken sum rule is by restricting the QCD analysis to the purely nonsinglet combination of the polarized parton distribution functions $\Delta q_{\mathrm{NS}}$. It is related to the structure functions, using Eq. (4),

$$
\begin{aligned}
& g_{1}^{p}\left(x, Q^{2}\right)-g_{1}^{n}\left(x, Q^{2}\right) \\
& \quad=\frac{1}{2}\left\langle e^{2}\right\rangle \int_{x}^{1} \frac{d y}{y}\left[C_{1, \mathrm{NS}}\left(\frac{x}{y}, \alpha_{s}(t)\right) \Delta q_{\mathrm{NS}}(y, t)\right],
\end{aligned}
$$

where $t=\ln \left(Q^{2} / \Lambda^{2}\right)$. The $Q^{2}$ dependence of $\Delta q_{\mathrm{NS}}$ is described by the DGLAP evolution equation for the nonsinglet combination [Eq. (8)] and is decoupled from the evolution of $\Delta \Sigma$ and $\Delta g$. Thus, having $g_{1}^{p}-g_{1}^{n}$ data points at different values of $Q^{2}$ allows us to determine $g_{A} / g_{V}$ by parametrizing only $\Delta q_{\mathrm{NS}}$ at an initial scale $Q_{i}^{2}$, evolving it, and fitting the parameters including $g_{A} / g_{V}$ to the data. The advantage of this method is that the analysis can be performed with fewer free parameters than the standard analysis presented in the previous sections. We use the parametrization

$$
\Delta q_{\mathrm{NS}}\left(x, Q^{2}\right)=\frac{3}{2}\left|\frac{g_{A}}{g_{V}}\right| N(\alpha, \beta) x^{\alpha}(1-x)^{\beta},
$$

with $\alpha, \beta$, and $g_{A} / g_{V}$ being the three free parameters of the fit. However, there is a disadvantage to this method. In order to evaluate the value of $g_{1}^{p}-g_{1}^{n}$ to be used in this fit, the values of the proton and neutron structure functions should be known ideally the same values of $x$ and $Q^{2}$. This is true only for SMC [1] and E143 [18-20] data. The SMC data points for $g_{1}^{p}$ and $g_{1}^{d}$ were combined as explained in Ref. [1]. The E143 data were treated similarly. In all we obtain 44 data points for $g_{1}^{\mathrm{NS}}$ (12 from SMC and 32 from E143). The general procedure of the analysis is the same as explained in
Sec. II C except that here it is done only with the nonsinglet parton distribution. The initial scale $Q_{i}^{2}=1 \mathrm{GeV}^{2}$ was used in this analysis as it was in the global PQCD analysis.

The values of the fitted parameters are given in Table IX. The result of the fit at $Q_{0}^{2}=5 \mathrm{GeV}^{2}$ is displayed in Fig. 10 . The data points evolved to the same $Q_{0}^{2}=5 \mathrm{GeV}^{2}$ are shown with their statistical errors. The bold line is the curve calculated using the best fit parameters. The area under this line corresponds to the Bjorken integral $\Gamma_{1}^{\mathrm{NS} \text {-fit }}$. The uncertainty band around this line shows the total uncertainty estimated from the experimental systematic and theoretical sources. The uncertainty (experimental systematic and that of theoretical origin) for the fitted value of $g_{A} / g_{V}$ was estimated. We get

$$
\left|\frac{g_{A}}{g_{V}}\right|=1.20_{-0.07}^{+0.08}(\text { stat })_{-0.12}^{+0.12}(\text { syst })_{-0.04}^{+0.10}(\text { th }) .
$$

At $Q_{0}^{2}=5 \mathrm{GeV}^{2}$ this value of $g_{A} / g_{V}$ corresponds to the Bjorken sum

$$
\Gamma_{1}^{p}-\Gamma_{1}^{n}=0.181_{-0.011}^{+0.012}(\mathrm{stat})_{-0.018}^{+0.018}(\mathrm{syst})_{-0.006}^{+0.015}(\mathrm{th})=0.181_{-0.021}^{+0.026}
$$

using Eq. (16) when evaluated at $\mathcal{O}\left(\alpha_{s}^{2}\right)$. The result for $g_{A} / g_{V}$ agrees well with the nominal value and with the results of the standard fit with $g_{A} / g_{V}$ as a free parameter (Eq. 17). Because of the smaller data set used the errors of ex-

TABLE IX. Best fit parameters for the $g_{1}^{\mathrm{NS}}$ fit with their statistical errors.

\begin{tabular}{cc}
\hline \hline Parameter & Value \\
\hline$\left|\frac{g_{A}}{g_{V}}\right|$ & $1.20_{-0.07}^{+0.08}$ \\
$\alpha$ & \\
$\beta$ & $0.20_{-0.12}^{+0.13}$ \\
$\chi^{2}$ & $1.42_{-0.36}^{+0.40}$ \\
$N_{\mathrm{DF}}$ & 52.4 \\
\hline \hline
\end{tabular}




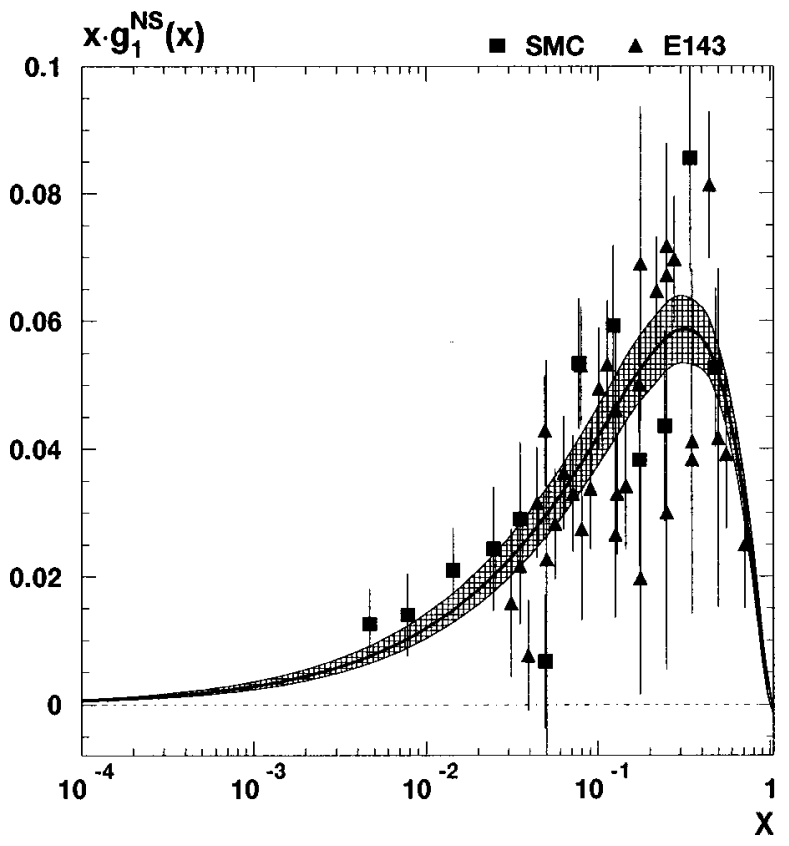

FIG. 10. The result of the best fit to $x g_{1}^{\mathrm{NS}}$ together with the data points used in the fit evolved to $Q^{2}=5 \mathrm{GeV}^{2}$. The error bars on the data points show statistical errors only, while the error band around the curve (cross hatch) represents the systematic uncertainty of the fit, including contributions from experimental systematic and theoretical sources.

perimental origin are significantly larger. However note that the theoretical error is slightly lower than in the case of the standard fit.

The contribution to the Bjorken sum from the measured $x$ region calculated from the data points and by integrating the fitted function are given in Table $\mathrm{X}$ in columns 4 and 5, respectively. They are given for combined $\mathrm{SMC}+\mathrm{E} 143$ data at $Q_{0}^{2}=5 \mathrm{GeV}^{2}$ as well as for SMC data at $Q_{0}^{2}=10 \mathrm{GeV}^{2}$. In both cases the integral over the measured $x$ range evaluated using the data and that evaluated using the best fit parameters agree within the statistical precision of the data. The high $x$ contribution to the integral makes little impact on the nonsinglet first moment. At both values of $Q_{0}^{2}$ the contributions to the integrals $\Gamma_{1}^{\mathrm{NS}}$ from the unmeasured low $x$ region are $\approx 5 \%$ of the total integral with small uncertainties. Hence we note that although the uncertainties in the first moments of the proton and neutron are large (Tables V and VII), the uncertainty in the Bjorken integral from this region is rather small.

\section{Comments on Bjorken sum rule determination}

In Section III C 1 we have presented a determination of the Bjorken sum rule, based on the final SMC data set and all other published data on $g_{1}$. The result was obtained in a NLO QCD analysis by directly fitting the value of $g_{A} / g_{V}$. This is our best determination of the Bjorken sum in a fully consistent way based on PQCD using the world data set.

The result we obtain is consistent with the expected value and we confirm the Bjorken sum rule with an accuracy of $\approx 10 \%$. It also agrees well with the results of the NLO QCD analysis of the E154 Collaboration [9]. Our estimate of the uncertainty is larger for the following reason: we have taken the view that the errors due to the factorization and renormalization scales and those due to $\alpha_{s}$ are uncorrelated where as they have treated them as correlated. If we follow their approach, the uncertainties become comparable.

The method used in Sec. III C 2 to test the Bjorken sum rule from $g_{1}^{\mathrm{NS}}$ is potentially very precise with regard to the theoretical uncertainty. It leads to a confirmation of the Bjorken sum rule at the level of $\approx 15 \%$. At present this method suffers from a limited statistical accuracy but it is expected to be more powerful once the very precise data on $g_{1}^{p}$ from E155 [36] become available and are combined with the existing data on $g_{1}^{n}$ from E154 [22].

\section{CONCLUSIONS AND SUMMARY}

We have performed a next-to-leading order PQCD analysis of the world data on polarized deep inelastic inclusive scattering, including new data from SMC. The results of the PQCD fit are used to evaluate contributions to the first moment of $g_{1}$ over the entire $x$ range. Consistent values of the singlet axial charge $a_{0}$ are obtained from the first moments and from the fit parameters.

The experimental data constrain the quark singlet and nonsinglet distributions rather well. This was tested using two different analysis programs. The polarized structure functions are equally well reproduced by fits in the $\overline{\mathrm{MS}}$ and

TABLE X. Integrals of the nonsinglet structure function in the measured and unmeasured $x$ ranges. Integrals are calculated using data (column 4) and using fit parameters (column 5) at $Q^{2}=5 \mathrm{GeV}^{2}$ using the SMC and E143 data, and at $Q_{0}^{2}=10 \mathrm{GeV}^{2}$ using only SMC data. The indicated uncertainties in the measured $x$ range are the statistical and systematic uncertainties, respectively.

\begin{tabular}{|c|c|c|c|c|}
\hline Data & $x$ range & $\begin{array}{c}Q_{0}^{2} \\
\mathrm{GeV}^{2}\end{array}$ & $\int_{x \min }^{x \max } g_{1}^{\mathrm{NS}}\left(Q_{0}^{2}\right)$ & $\int_{x \min }^{x \max } g_{1}^{\text {NS-fit }}\left(Q_{0}^{2}\right)$ \\
\hline & $0 \rightarrow 0.003$ & 5 & & 0.009 \\
\hline \multirow[t]{3}{*}{$\mathrm{SMC}+\mathrm{E} 143$} & $0.003 \rightarrow 0.8$ & 5 & $0.174 \pm 0.011 \pm 0.013$ & 0.170 \\
\hline & $0.8 \rightarrow 1.0$ & 5 & & 0.002 \\
\hline & $0 \rightarrow 0.003$ & 10 & & 0.010 \\
\hline \multirow[t]{2}{*}{ SMC } & $0.003 \rightarrow 0.7$ & 10 & $0.184 \pm 0.016 \pm 0.014$ & 0.169 \\
\hline & $0.7 \rightarrow 1.0$ & 10 & & 0.004 \\
\hline
\end{tabular}


the $\mathrm{AB}$ factorization schemes, although the shapes of the singlet distributions are found to be different. The singlet and nonsinglet quark distributions are well determined, while the gluon distribution is only poorly constrained by the fits. The gluon first moment is found to be positive but has an error of the order of $100 \%$ of its value. The singlet axial charge is found to be $\approx 1 / 3$ of the value expected from the naive QPM.

Inclusion of the strong coupling constant $\alpha_{s}$ as a free parameter in the fit results in a value for $\alpha_{s}$ in excellent agreement with the one obtained from the observation of scaling violations in unpolarized DIS data. However, this determination based on $g_{1}$ also involves $F_{2}$ and hence is not independent of the determination of $\alpha_{s}$ from $F_{2}$.

The Bjorken sum rule has been tested in two different ways: in a global PQCD analysis and in an analysis restricted to the non-singlet part of $g_{1}$ performed using a subset of the available data. In both cases $\left|g_{A} / g_{V}\right|$ was left as a free parameter of the fit. The sum rule is found to be verified in both cases, within an accuracy of about $10 \%$ for the global fit and $15 \%$ for the non-singlet fit.

In the near future, the additional high precision data from SLAC E155 are expected to improve the accuracy of the QCD fit. However due to the absence of data in the low $x$ region, contribution to the first moment from this region is expected to be the largest source of uncertainty. Improved determinations of the polarized gluon distribution will be obtained by dedicated experiments, e.g., COMPASS [37] at
CERN and PHENIX and STAR experiments at the RHIC Spin [38]. Measurements of the spin structure function in the presently inaccessible low $x$ region using the HERA polarized collider [39] will provide crucial information on the low $x$ behavior of $g_{1}$ and also allow access to the polarized gluon distribution in that region.

\section{ACKNOWLEDGMENTS}

We thank G. Altarelli, R. D. Ball, S. Forte, T. Gehrmann, G. Ridolfi, and W. Vogelsang for many interesting discussions. Special thanks are due to R. D. Ball, S. Forte, and G. Ridolfi for providing us with the PQCD analysis code. This work was supported by Bundesministerium für Bildung, Wissenschaft, Forschung und Technologie, partially supported by TUBITAK and the Center for Turkish-Balkan Physics Research and Application (Bogziçi University), supported by the U.S. Department of Energy, the U.S. National Science Foundation, Monbusho Grant-in-Aid for Science Research (International Scientific Research Program and Specially Promoted Research), the National Science Foundation (NWO) of the Netherlands, the Commisariat à l'Energie Atomique, Comision Interministerial de Ciencia y Tecnologia and Xunta de Galicia, the Israel Science Foundation, and Polish State Committee for Scientific Research (KBN) Grant No. 2/P03B/081/14.
[1] SMC Collaboration, B. Adeva et al. preceding paper, Phys. Rev. D 58, 112001 (1998).

[2] J. Ellis and R. L. Jaffe, Phys. Rev. D 9, 1444 (1974); 10, 1669 (1974).

[3] G. Altarelli and G. G. Ross, Phys. Lett. B 212, 391 (1988); A. V. Efremov and O. V. Teryaev, JINR Report No. E2-88-287, Dubna, 1988; R. D. Carlitz, J. C. Collins, and A. H. Mueller, Phys. Lett. B 214, 229 (1988).

[4] J. D. Bjorken, Phys. Rev. 148, 1467 (1966); Phys. Rev. D 1, 1376 (1970).

[5] M. Glück, E. Reya, M. Stratmann, and W. Vogelsang, Phys. Rev. D 53, 4775 (1996).

[6] R. D. Ball, S. Forte, and G. Ridolfi, Phys. Lett. B 378, 255 (1996).

[7] T. Gehrmann and W. J. Stirling, Z. Phys. C 65, 461 (1995); Phys. Rev. D 53, 6100 (1996).

[8] G. Altarelli, R. D. Ball, S. Forte, and G. Ridolfi, Nucl. Phys. B496, 337 (1997).

[9] E154 Collaboration, K. Abe et al., Phys. Lett. B 405, 180 (1997)

[10] SMC Collaboration, D. Adams et al., Phys. Lett. B 396, 338 (1997).

[11] SMC Collaboration, B. Adeva et al., Phys. Lett. B 412, 414 (1997).

[12] SMC Collaboration, D. Adams et al., Phys. Rev. D 56, 5330 (1997)

[13] NMC Collaboration, M. Arneodo et al., Nucl. Phys. B483, 3 (1997).
[14] L. W. Whitlow et al., Nucl. Phys. B282, 475 (1992).

[15] H.-Y. Cheng, Chin. J. Phys. 35, 25 (1996).

[16] V. W. Hughes and J. Kuti, Annu. Rev. Nucl. Part. Sci. 33, 611 (1983); T. Pussieux and R. Windmolders, in Internal Spin Structure of the Nucleon, edited by V. W. Hughes and C. Cavata (World Scientific, Singapore, 1995), p. 212.

[17] EMC Collaboration, J. Ashman et al., Phys. Lett. B 206, 364 (1988); Nucl. Phys. B328, 1 (1989).

[18] E143 Collaboration, K. Abe et al., Phys. Rev. Lett. 74, 346 (1995).

[19] E143 Collaboration, K. Abe et al., Phys. Rev. Lett. 75, 25 (1995).

[20] E143 Collaboration, K. Abe et al., Phys. Lett. B 364, 61 (1995).

[21] E142 Collaboration, P. Anthony et al., Phys. Rev. D 54, 6620 (1996).

[22] E154 Collaboration, K. Abe et al., Phys. Rev. Lett. 79, 26 (1997).

[23] HERMES Collaboration, K. Ackerstaff et al., Phys. Lett. B 404, 383 (1997).

[24] V. N. Gribov and L. N. Lipatov, Sov. J. Nucl. Phys. 15, 438 (1972); 15, 675 (1972); G. Altarelli and G. Parisi, Nucl. Phys. B126, 298 (1977); Yu. L. Dokshitzer, Sov. Phys. JETP 46, 641 (1977).

[25] J. Kodaira et al., Phys. Rev. D 20, 627 (1979); J. Kodaira, Nucl. Phys. B165, 129 (1980).

[26] R. Mertig and W. L. van Neerven, Z. Phys. C 70, 637 (1996); 
W. Vogelsang, Nucl. Phys. B475, 47 (1996).

[27] R. G. Roberts, The Structure of the Proton, Cambridge Monographs on Mathematical Physics (Cambridge University Press, Cambridge, England, 1990).

[28] Particle Data Group, R. M. Barnett et al., Phys. Rev. D 54, 1 (1996).

[29] F. E. Close and R. G. Roberts, Phys. Lett. B 316, 165 (1993).

[30] S. J. Brodsky, M. Burkardt, and I. Schmidt, Nucl. Phys. B441, 197 (1995).

[31] D. Fasching, Ph.D thesis, Northwestern University, 1996; hep-ph/9610261.

[32] NMC, M. Arneodo et al., Phys. Lett. B 309, 222 (1993); M. Virchaux and A. Milsztajn, ibid. 274, 221 (1992); CCFR Collaboration, W. G. Seligman et al., Phys. Rev. Lett. 79, 1213 (1997); R. D. Ball and S. Forte, in Proceedings of DIS96, Rome, 1996 (unpublished).

[33] J. Saborido, Ph.D. thesis, University of Santiago de Compostela, 1995.
[34] E. P. Sichtermann, Ph.D. thesis, Free University of Amsterdam, 1998.

[35] S. A. Larin, F. V. Tkachev, and J. A. M. Vermaseren, Phys. Rev. Lett. 66, 862 (1991); S. A. Larin and J. A. M. Vermaseren, Phys. Lett. B 259, 345 (1991).

[36] E155 Collaboration, e.g., C. Young, in Deep Inelastic Scattering off Polarized Targets, Proceedings of the Workshop, Zeuthen, Germany, 1997, edited by J. Blümlein et al. (DESY Report No. 97-200, Hamburg, 1997).

[37] COMPASS proposal, CERN/SPSLC/P297, 1996.

[38] Y. I. Makdisi, in Proceedings of 12th International Symposium on High Energy Spin Physics, Amsterdam 1996, edited by C. W. de Jager et al. (World Scientific, Singapore, 1997), p. 107.

[39] A. Deshpande et al., in Physics with Polarized Protons at HERA, Proceedings of the Workshop, Hamburg, Germany, 1997, edited by A. De Roeck and T. Gehrmann (DESY, Hamburg, 1998), p. 26; G. Rädel et al., ibid., p. 54; A. De Roeck et al., Eur. Phys. J. C (to be published), hep-ph/9801300. 